Universidade de São Paulo

Instituto de Física

SBI-IFUSP

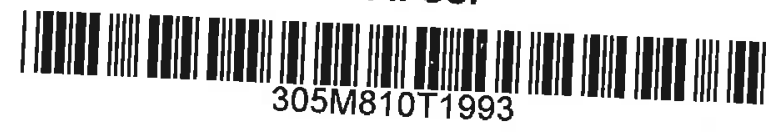

\title{
UM ESTUDO DO MÉTODO DE MONTE CARLO DE CAMPO MÉDIO
}

Dissertação de Mestrado apresentada ao Instituto de Física da Universidade de São Paulo

Mrimitas:

Orientador: Prof. Dr/Sílvio Roberto Azevedo Salinas

Autor: Eduardo Fontes Henriques
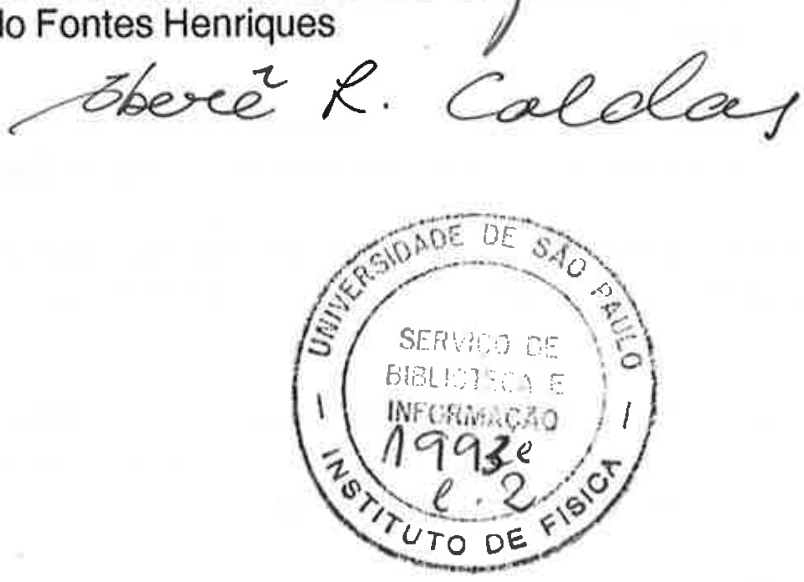


\section{FICHA CATALOGRAFICA}

Preparada pelo Serviço de Biblioteca e Informação do Instituto de Fisica da Universidade de são Paulo

Henriques, Eduardo Fontes

Um estudo do método de Monte-Carlo de campo médio. São Paulo, 1992.

Dissertação (Mestrado) - Universidade de São Paulo. Instituto de Física. Departamento de Física Experimental.

Área de Concentração: Física do Estado Sólido Salinas

Orientador: Profo Dro Silvio Roberto de Azevedo

Unitermos: 1. Transições de fases; 2. Método de Monte-Carlo; 3. Campo medio; 4. Modelo de Ising ferromagnético; 5. Modelo de Blume-Capel.

$\mathrm{USP} / \mathrm{IF} / \mathrm{SBI}-41 / 92$ 
A meus pais; Liège e Ivo 


\section{Resumo}

Utilizamos o método de Monte Carlo de campo médio, recentemente proposto por Netz e Berker, para estudar o comportamento termodinâmico dos modelos de Ising e de Blume-Capel numa rede quadrada. Esse método mistura conceitos de amostragem aleatória (Monte Carlo) com equações de campo médio usual. Seus autores afirmam que o método pode permitir representações de diagramas de fase com amostragens muito menores do que as usadas nas simulações de Monte Carlo convencionais e com a eliminação de certas consequências indesejáveis da aplicação das equações de consistência de campo médio. Entretanto, não observamos, pelo menos nos modelos que foram estudados, uma tendência clara de redução das amostragens (número de passos de Monte Carlo) em relação a simulações computacionais pelos métodos conhecidos. Além disso os nossos cálculos apontam na direção de uma grande semelhança com os resultados usuais de uma aproximação de Bethe-Peierls. Estes problemas devem ser somados ao fato de não haver uma boa explicação para o mecanismo do método de Netz e Berker, dada a dificuldade de estudar a dinâmica em que ele se baseia. 


\section{Abstract}

We have used the method of Monte Carlo Mean Field, recently proposed by Netz and Berker, to study the thermodynamic behavior of the Ising and Blume-Capel models on square lattices. This method merges concepts of stochastic sampling (Monte Carlo) with the usual mean-field equations. Their authors claim that the method permits representations of phase diagrams with much less samplin gs than those used in conventional Monte Carlo simulations, eliminating also certain undesirable consequences of the application of the mean - field consistency equations. However, we haven ' $t$ observed, at least in the models we have studied, a clear tendency of a reduction of the samplings ( number of Monte Carlo steps) compared with computational simulations by other known methods. Also, our calculations point to great resemblances with usual results given by Bethe-Peierls approximations. To these problems, we must add the fact that there is no good expalnation for the machinery of Netz and Berker's method, given the difficulty of sudying the stochastic dynamics on which it is based. 


\section{Agradecimentos}

Ao professor Newton Bernardes, que com complacência e generosidade me introduziu aos assuntos da Termodinâmica e da Mecânica Estatística. Sem sua contribuição qualquer desenvolvimento posterior teria sido substancialmente mais difícil.

Aos professores S.R.A. Salinas e Vera B. Henriques, que num momento difícil aceitaram um trabalho paciente de co-orientação.

Aos meus colegas e amigos Luís Fernandez Lopez, com quem estudamos e discutimos boa parte deste trabalho, e Niels Fontes Lima, pelas suas observações sempre úteis.

Aos professores Carlos Eugênio I. Carneiro e Mário J. de Oliveira, cujos conselhos foram de grande ajuda.

A todos os colegas do Departamento ( especialmente Nestor Oiwa ) que tiveram a boa vontade de me ajudar nos problemas computacionais.

Ao CNPQ e à Comissão de Pós Graduação do IFUSP, pela bolsa que tornou possível minha dedicação integral a esse trabalho.

A Carmen, que pacientemente realizou o trabalho de editoração desta tese, aceitando além disso muitas horas sem a minha presença e mantendo sempre seu apoio decidido.

À família Nunes, particularmente ao Pedro, pela gentileza em nos emprestar seu computador para a edição deste trabalho. 
I - Introdução

II - O Algoritmo de Netz e Berker e sua aplicação ao Modelo de Ising.

II.1 - A aproximação de campo médio para o Modelo de Ising 3

II.2 - O Algoritmo de Netz e Berker 7

III - A aplicação do algoritmo sobre a rede quadrada feromagnética de Ising.

III. 1 - O desenvolvimento do algoritmo 12

III.2 - Resultados qualitativos para a magnetização por spin

III.3 - A análise de erros pelo método de ineficiência estatística 16

III.4 - Apresentação de resultados a campo nulo com base em cálculos de energia por spin.

IV - A aplicação do algoritmo de Netz e Berker ao Modelo de Blume-Capel.

IV.1 - Apresentação do modelo de Blume-Capel

IV.2 - A obtenção da prescrição de Netz e Berker para o modelo

de Blume-Capel .27

IV.3 - A implementação do algoritmo

IV.4 - A forma geral das curvas de magnetização e $Q=\left\langle\left(\sum_{i} s_{i}^{2}\right) /(\mathrm{N} \mathrm{x} \mathrm{N})\right\rangle$ em redes quadradas. .30

IV.5 - A localização dos pontos de transição e o levantamento do diagrama de fases .33

V - Conclusão .36

Apêndice 1 . .37

Apêndice 2 38

Apêndice 3 42

Apêndice 4 48

Referências .50 


\section{I - Introdução}

Os métodos de campo médio, em seus sentidos variados (Bragg-Williams, Curie-Weiss etc), são de fundamental importância em Mecânica Estatística de sistemas de partículas interagentes pois, em geral, mesmo para os modelos mais simples que existem para representar tais sistemas, as dificuldades matemáticas que surgem no cálculo das funções de partição são enormes ou mesmo insuperáveis. Assim, as abordagens de campo médio, através de uma deformação no problema original, são muito utilizadas como uma primeira forma de obter informações sobre um determinado modelo estatístico.

A deformação acima mencionada não deve levar à conclusão de que a aplicação desses métodos implica necessariamente resultados grosseiros. Na verdade, foi com um protótipo desses métodos que Van der Waals, em 1873, foi capaz de mostrar, pela primeira vez com sucesso, a influência que deveriam ter os sistemas de moléculas interagentes na descrição estatística dos fenômenos de transição de fases e de criticalidade (Thompson, 1988, Brush, 1983). Podemos, nesse particular, lembrar a concordância com dados experimentais a que conduz a lei dos estados correspondentes, obedecida pela equação de Van der Waals (Stanley,1971). Um outro exemplo de teoria de campo médio de grande valor heurístico e histórico foi a teoria do campo molecular de P. Weiss, criada em 1907 para acomodar resultados quantitativos sobre o fenômeno do ferromagnetismo que já eram conhecidos desde o trabalho de P. Curie de 1898 (Brush, 1983).

Entretanto, apesar desses fatos, os cálculos de campo médio não são capazes de reproduzir, em geral, as propriedades mais importantes da matéria, particularmente em seu estado crítico. Além disso certos fatores, como a dimensionalidade da rede cristalina ou o alcance das interações, jogam um papel secundário nessa aproximação. Em algumas ocasiões, somos levados a resultados que sabemos não corresponder em absoluto às características do modelo que estamos tratando. $O$ exemplo mais conhecido desse fato é um cálculo de campo médio para o modelo de Lenz-Ising sobre uma cadeia linear, que conduz ao resultado, incompatível com o cálculo exato, de ordenamento abaixo de uma temperatura de Curie não nula.

Existem várias formas de superar ou melhorar as deficiências dos métodos de campo médio. Podemos, por exemplo, mudar totalmente o enfoque sobre o problema através de simulações computacionais, como a de Metrópolis, ou por aplicação de outras técnicas sofisticadas de Mecânica Estatística. Há, além disso, num campo intermediário, maneiras de refinar o espírito das aproximaçōes de campo médio que melhoram os resultados obtidos. Como exemplo deste último tipo de abordagem podemos destacar a aproximação de Bethe-Peierls (Pathria, 1972) que, entre outros resultados, remove da aproximação de campo médio do modelo de Ising linear a existência de uma temperatura de Curie.

Roland R. Netz e A. Nihat Berker propuseram um método, na linha das aproximações de campo médio, que utiliza conceitos de Monte Carlo através de um algoritmo computacional. Segundo esses autores, poderiam ser removidos alguns problemas das soluções usuais de campo médio com amostragems muito menores do que as usadas nos tratamentos convencionais de Monte Carlo (Netz, 1991 a, b e c). Seu interesse central são os modelos frustados e a primeira aplicação proposta foi sobre uma rede antiferromagnética triangular de Ising, que não possui nenhum ordenamento em temperaturas finitas, em contradição com o resultado de campo médio, de ordenamento em três subredes abaixo de uma certa temperatura de Neél. Como Netz e Berker entendem que o algoritmo é de aplicação geral em Mecânica Estatística, decidimos analisar as principais características deste método. Escolhemos então um sistema mais conhecido, que é o modelo de Ising na rede quadrada ferromagnética com simetria translacional. Além desse sistema, para o qual apresentaremos resultados quantitativos da aplicação do algoritmo de Netz e Berker (que chamaremos NB) também estudamos o Modelo de Blume-Capel, que é capaz de sofrer transições tanto de primeira como de segunda or- 
dem. Procuramos verificar se o algoritmo de NB é capaz de distinguir a ordem de uma transição e'de oferecer uma representação, ao menos qualitativa, do diagrama de fases do modelo de Blume-Capel.

Antes de iniciarmos a apresentação do algoritmo de NB, revisaremos sucintamente a aproximação de campo médio obtida através da desigualdade de Bogoliubov, ou método variacional (Callen,1985), que usaremos como um guia tanto para explicar o algoritmo quanto para estendê-lo a modelos de spin 1 (Blume-Capel). Essa escolha é nossa, isto é, esse caminho nāo é usado pelos autores do algoritmo, mas pensamos que pode nos ajudar a fixar nossa notação e, dessa forma, a entender a proposta. Posteriormente, iremos introduzir o algoritmo e a interpretação dada pelos seus autores. Nos capítulos finais mostraremos os resultados obtidos para os modelos propostos e concluiremos com uma discusșão desses resultados, razão pela qual esses capítulos formam a parte central desse trabalho . 


\section{II - O Algoritmo de Netz e Berker e sua Aplicação ao Modelo de Ising.}

\section{II.1 - A Aproximação de Campo Médio para o Modelo de Ising.}

Suponhamos que nos é apresentado o problema de se aproximar as propriedades termodinâmicas de um sistema cuja hamiltoniana $\mathrm{H}$ torne muito difícil ou impossível o cálculo da função de partição. O princípio variacional de Bogoliubov, de validade geral, nos permite afirmar que, sendo dada uma hamiltoniana de teste $\mathrm{H}_{0}$, a função de Gibbs, $G$, representativa do sistema em questão, guarda com o sistema representado por $\mathrm{H}_{\circ}$ a seguinte relação:

$$
\begin{aligned}
& G \leq G_{o}+\left\langle\mathrm{H}-\mathrm{H}_{0}\right\rangle_{o}=\Phi \\
& \text { com } \\
& G_{o}=-\frac{1}{\beta} \ln \sum_{a_{o}} \exp \left(-\beta E_{a_{o}}\right),
\end{aligned}
$$

em que $a_{o}$ representa um auto-estado de $H_{0}$ de energia $E_{a_{o}}$ e onde $<H-H_{0}>$ representa a média térmica de $\mathrm{H}-\mathrm{H}_{0}$ na distribuição estatística gerada por $\mathrm{H}_{\mathrm{o}}$. Esse teorema, cuja demonstração se encontra nos manuais de Mecânica Estatística ( Callen, 1985 ), nos dá uma das formas de chegarmos ao tratamento de campo médio. Mostraremos isso no caso de um Modelo de Ising isotrópico.

Consideramos uma rede de $\mathrm{N}^{\mathrm{d}}$ sítios (de posições indicadas sempre pelas letras $i, j$, etc.) nos quais colocamos sinais +1 ou -1 (indicados por $\sigma_{i}= \pm 1, \sigma_{j}= \pm 1$,etc). A energia de uma configuração $\{\sigma\}$ desses "spins" na rede será dada por

$$
H(\{\sigma\}, h)=-J \sum_{<i j>} \sigma_{i} \sigma_{j}-h \sum_{i} \sigma_{i}
$$

onde o primeiro termo indica uma soma sobre pares de vizinhos mais próximos e o segundo termo representa a interação com um campo externo $h$ em unidades convenientes. $O$ caso $J>O$ representa um ferromagneto e $J<0$ um antiferromagneto.

No espírito dos princípios variacionais, a aproximação de campo médio pode ser obtida pelo uso da hamiltoniana de teste

$$
H_{\circ}=-J \sum_{i} H_{i} \sigma_{i}-h \sum_{i} \sigma_{i}
$$

Veremos como o uso de "campos locais $H_{i}$ "nos leva às expressões usuais de campo médio".

A distribuição estatística de $H_{0}$ será dada por

$$
\begin{aligned}
Z_{o} & =\sum_{\{\sigma\}} \exp \left(\beta J \sum_{i} H_{i} \sigma_{i}+\beta h \sum_{i} \sigma_{i}\right) \\
& =\prod_{i} 2 \cosh \left[\beta J\left(H_{i}+h^{\prime}\right)\right] \quad\left(h=J h^{\prime}\right) .
\end{aligned}
$$

Segue-se disso que o limite superior da função de Gibbs será dado por 


$$
\begin{aligned}
\Phi\left(\left\{H_{i}\right\}\right)=G_{o}+\left\langle H-H_{o}\right\rangle_{o} \\
\left.=-\frac{1}{\beta} \sum_{i} \ln \left\{2 \cosh \left[\beta J\left(H_{i}+h^{\prime}\right)\right]\right\}-J \sum_{<i j\rangle}\left\langle\sigma_{i} \sigma_{j}\right\rangle_{o}+\sum_{i} J H_{i}<\sigma_{i}\right\rangle_{o} \\
=-\frac{1}{\beta} \sum_{i} \ln \left\{2 \cosh \left[\beta J\left(H_{i}+h^{\prime}\right)\right]\right\}-J \sum_{<i j\rangle} \tanh \left[\beta J\left(H_{i}+h^{\prime}\right)\right] \tanh \left[\beta J\left(H_{j}+h^{\prime}\right)\right] \\
+\sum_{i} J H_{i} \tanh \left[\beta J\left(H_{i}+h^{\prime}\right)\right],
\end{aligned}
$$

uma vez que $\left\langle\sigma_{i}\right\rangle_{0}=\tanh \left[\beta J\left(H_{i}+h^{\prime}\right)\right]$ e que a forma de $H_{0}$ implica descorrelação de spins (independência estatística dos spins nos sítios). A condição de melhor aproximação para a função de Gibbs é obtida mimimizando $\Phi\left(\left\{H_{i}\right\}\right)$ sobre o conjunto $\left\{H_{i}\right\}$. Tem-se então

$$
\begin{aligned}
\frac{\partial \Phi\left(\left\{H_{i}\right\}\right)}{\partial H_{i}}= & -\frac{1}{\beta} \frac{\partial}{\partial H_{i}}\left\{\ln \left[2 \cosh \left(\beta J\left(H_{i}+h^{\prime}\right)\right]\right\}\right. \\
& -\left\{J \sum_{j} \tanh \left[\beta J\left(H_{j}+h^{\prime}\right)\right]\right\} \frac{\partial}{\partial H_{i}} \tanh \left[\beta J\left(H_{i}+h^{\prime}\right)\right] \\
& +\frac{\partial}{\partial H_{i}}\left\{J H_{i} \tanh \left[\beta J\left(H_{i}+h^{\prime}\right)\right]\right\}=0,
\end{aligned}
$$

de onde vem que

$$
J H_{i}-J \sum_{i} \tanh \left[\beta J\left(H_{j}+h^{\prime}\right)\right]=0,
$$

ou seja,

$$
H_{i}=\sum_{j}\left\langle\sigma_{j}\right\rangle_{0},
$$

onde a soma é para os primeiros vizinhos do sítio $i$, indicados por $j$. Portanto, $\left\{H_{i}\right\}$, ou equivalentemente $\left.\left\{<\sigma_{i}\right\rangle_{0}\right\}$, será determinado por

$$
\left.<\sigma\rangle_{0}=\tanh \left[\beta J\left(\sum_{j}<\sigma_{j}\right\rangle_{0}+h^{\prime}\right)\right],
$$

para $i=1,2, \quad, \mathrm{~N}^{\mathrm{d}}$, que são as equações de consistência para os $\mathrm{N}^{\mathrm{d}}$ valores das magnetizações locais $\left\langle\sigma_{i}>\right.$. Em geral utilizamos condições periódicas de contorno. No limite termodinâmico, $\mathrm{N} \rightarrow \infty$, no entanto, as condiçōes de contorno não devem influenciar os resultados finais. Vejamos rapidamente duas aplicações conhecidas desse sistema de equações.

No caso ferromagnético, por exemplo, dada a simetria translacional, podemos fazer $\left\langle\sigma_{i}\right\rangle=m_{0}$ em todos os sítios. Dado o número de coordenação da rede, $q$, e condições periódicas de contorno, teremos a redução do sistema a uma única equação:

$$
m_{0}=\tanh \left[\beta J\left(q m_{0}+h^{\prime}\right)\right](J>0) .
$$

Essa relação já nos mostra a equivalência do que fizemos acima com os tratamentos de campo médio do tipo "Curie-Weiss" (Thompson, 1988) ou Bragg-Williams (Pathria, 1972). Obtemos a temperatura crítica tomando principalmente $h^{\prime} \rightarrow 0$ e observando que a equação

$$
m_{0}=\tanh \left(\beta J q m_{0}\right)
$$

só possuirá a solução nula caso 
$\beta_{c} J q<1$

ou seja, para temperaturas maiores do que

$$
T_{c}=\frac{J q}{k_{B}},
$$

posssuindo também soluções não-nulas para $T<T_{c}$ (iguais em módulo e de sinais contrários), indicando $o$ ordenamento nessa região.

No caso de redes antiferromagnéticas $(J<0)$, o procedimento mais indicado é a subdivisão em subredes. Assim, por exemplo, no caso de uma rede quadrada, dividimos a rede em duas subredes A e B e o sistema de equações ficará reduzido a

$$
\begin{aligned}
& m_{A}=\tanh \left[\beta J\left(q m_{B}+h^{\prime}\right)\right] \\
& m_{B}=\tanh \left[\beta J\left(q m_{A}+h^{\prime}\right)\right] .
\end{aligned}
$$

No caso $h \rightarrow 0$, as soluções ficam sendo do tipo $M 0=m_{A}=-m_{B}$, com $M 0 \neq 0$ abaixo da temperatura de Neél $T_{N}=-J q / k_{B}$, indicando um ordenamento antiferromagnético a baixas temperaturas.

Mais acima dissemos que na aproximação de campo médio os valores de $<\sigma_{i}>$ são obtidos pelos próprios valores de $<\sigma_{i}>0$. Para mostrar que isso se verifica podemos modificar um pouco a hamiltoniana de Ising de forma a considerar campos externos locais $h_{i}$, diferentes a princípio, em cada um dos sítios da rede. A magnetização local no sítio $k$ será, por definição,

$$
\begin{aligned}
m_{k}=\left\langle\sigma_{k}>\right. & =-\frac{1}{J} \frac{\partial G\left(T_{i}\left\{h_{i}^{\prime}\right\}\right)}{\partial h^{\prime} k} \\
& =-\frac{1}{J} \frac{\partial}{\partial h^{\prime} k}\left[-\frac{1}{\beta} \ln \sum_{\{\sigma\}} e^{\beta J \sum_{<i j>} \sigma i \sigma j+\beta J \sum_{i}{h^{\prime}}_{i} \sigma i}\right] .
\end{aligned}
$$

Na aproximação de campo médio $G\left(T\left\{h^{\prime} i\right\}\right)$ é calculado a partir de $\Phi\left(\left\{H_{i}\right\}\right)$ onde, agora, $\left\{H_{i}\right\}$ está fixado pelas equações de consistência na forma

$$
H_{i}=\sum_{j} \tanh \left[\beta J\left(H_{j}+h^{\prime} i\right)\right] .
$$

Esse conjunto de equações implicará soluções onde todos os campos $H_{i}$ serão funções de todos os campos $h^{\prime} i$. Teremos então

$$
\begin{gathered}
m_{k}=-\frac{1}{J} \frac{\partial}{\partial h^{\prime}{ }_{k}}\left\{-\frac{1}{\beta} \sum_{i} \ln \left\{2 \cosh \left[\beta J\left(H_{i}+h^{\prime}{ }_{i}\right)\right]\right\}\right. \\
\left.-J \sum_{<i j>} \tanh \left[\beta J\left(H_{i}+h^{\prime}{ }_{i}\right)\right] \tanh \left[\beta J\left(H_{j}+h^{\prime}{ }_{j}\right)\right]+J \sum_{i} H_{i} \tanh \left[\beta J\left(H_{j}+h^{\prime}{ }_{i}\right)\right]\right\} \\
=\tanh \left[\beta J\left(H_{k}+h^{\prime}{ }_{k}\right)\right]+\frac{\partial}{\partial h^{\prime}{ }_{k}} \sum_{<i j>} \tanh \left[\beta J\left(H_{i}+h^{\prime} i\right)\right] \tanh \left[\beta J\left(H_{j}+h^{\prime}{ }_{j}\right)\right] \\
-\sum_{i} H_{i} \frac{\partial}{\partial h^{\prime}{ }_{k}}\left\{\tanh \left[\beta J\left(H_{i}+h^{\prime} i\right)\right]\right\}
\end{gathered}
$$

Em virtude das equações de consistência teremos

$$
m_{k}=\tanh \left[\beta J\left(H_{k}+h^{\prime} k\right)\right]+\sum_{<i j>} \tanh \left[\beta J\left(H_{i}+h_{i}^{\prime}\right)\right] \frac{\partial}{\partial h^{\prime} k}\left\{\tanh \left[\beta J\left(H_{j}+h^{\prime} j\right)\right]\right\}
$$


$+\sum_{<i j>} \frac{\partial}{\partial h^{\prime}{ }_{k}}\left\{\tanh \left[\beta J\left(H_{i}+h^{\prime}{ }_{i}\right)\right]\right\} \tanh \left[\beta J\left(H_{j}+h^{\prime}{ }_{j}\right)\right]$

$-\sum_{i} \sum_{j} \tanh \left[\beta J\left(H_{j}+h^{\prime}{ }_{j}\right)\right] \frac{\partial}{\partial h^{\prime}{ }_{j}}\left\{\tanh \left[\beta J\left(H_{i}+h^{\prime}{ }_{i}\right)\right]\right\}$

$=\tanh \left[\beta J\left(H_{k}+h^{\prime}{ }_{k}\right)\right]+\sum_{i} \sum_{j} \tanh \left[\beta J\left(H_{i}+h^{\prime}{ }_{i}\right)\right] \frac{\partial}{\partial h^{\prime}{ }_{k}}\left\{\tanh \left[\beta J\left(H_{j}+h^{\prime}{ }_{j}\right)\right]\right\}$

$-\sum_{i} \sum_{j} \tanh \left[\beta J\left(H_{i}+h^{\prime}{ }_{i}\right)\right] \frac{\partial}{\partial h^{\prime}{ }_{k}}\left\{\tanh \left[\beta J\left(H_{j}+h^{\prime}{ }_{j}\right)\right\}\right.$

$$
=\tanh \left[\beta J\left(H_{k}+h^{\prime} k\right)\right]=\left\langle\sigma_{k}\right\rangle_{0},
$$

que é o que desejávamos mostrar. Em outras palavras, a relação acima nos diz que a aproximação para a magnetização local é obtida a partir de $\mathrm{H}_{0}$ com os campos fixados pela condição de extremização de $\Phi\left(\left\{H_{i}\right\}\right)$.

Para finalizar, é importante ressaltar que, quando há soluções múltiplas para as equações de consistência, devemos tomar o conjunto de soluções que correspondem ao mínimo absoluto de $\Phi\left(\left\{H_{i}\right\}\right)$. É desta forma que podemos mostrar que existe um estado ordenado abaixo de $T_{c}$ ou $T_{\mathrm{N}}$ nos dois exemplos que discutimos mais acima. 


\section{II.2 - O Algoritmo de Netz e Berker}

Vamos fixar um pouco nossa atenção sobre o sistema de equações de campo médio

$$
<\sigma_{i}>=\tanh \left[\beta J\left(\sum_{j}<\sigma_{j}>+h^{\prime}\right)\right],
$$

onde a soma em $j$ é sobre os primeiros visinhos de sítio $i$ e $h^{\prime}=h / J$. Na aproximação de campo médio, a média térmica do spin num sítio é dada pelo campo externo e por um campo efetivo de troca, $\left.\sum_{j}<\sigma_{j}\right\rangle$. Esta expressão pode ser tomada como uma aproximação para uma relação mais geral (redemonstrada no Apêndice 1) e conhecida na literatura,

$$
\left.<\sigma_{i}\right\rangle_{b}=\left\langle\tanh \left[\beta J\left(\sum_{j} \sigma_{j}+h^{\prime}\right)\right]\right\rangle_{b}
$$

onde $<\ldots>b$ representa a média térmica na própria distribuição de Boltzmann.

Segundo o enfoque de Netz e Berker, é na aproximação introduzida nas equações acima que está um dos problemas com os cálculos de campo médio, uma vez que, em suas palavras, as equações de campo médio estariam a nos dizer que um sítio $i$ sente um campo efetivo de troca construído pelos valores médios dos spins de seus primeiros vizinhos, enquanto que sabemos que o campo de troca real é dado pela soma desses spins, podendo inclusive se anular para qualquer temperatura.

Feitas as considerações acima, Netz e Berker propõe um algoritmo, chamado "teoria de Monte Carlo de Campo Médio" ou "teoria de Campo Médio de " spin duro"'", para levar em conta o campo de troca real num dado sítio. Esse algoritmo consiste em atualizar a magnetização local em um sítio, $m_{i}$, por meio de equações, formalmente idênticas às equações de campo médio, do tipo, $m_{i}=\tanh \left[\beta J\left(H_{i}+h^{\prime}\right)\right]$, mas onde o campo de troca $H_{i}$ é construído por $H_{i}=\sum_{j} \sigma_{j}$, com $\sigma_{j}= \pm 1$, determinado por uma amostragem estocástica, nomeadamente, pelos sinais de $m_{j}-r$, onde $r$ é um número aleatório entre -1 e +1 .

Vamos tornar mais claro o algoritmo por meio de um exemplo. Observemos, antes disso, que a prescrição para colocarmos no sítio $j$, vizinho de um sítio $i$ onde estamos fazendo uma atualização, um spin de um determinado sinal, é equivalente ao uso de uma probabilidade "de transição"

$$
\omega_{j}\left(\sigma_{j}\right)=\frac{1}{2}\left(1+\sigma_{j} m_{j}\right),
$$

onde $\omega_{j}\left(\sigma_{j}\right)$ é a probabilidade do spin no sítio $j$ adquirir o valor $\sigma_{j}$. Vejamos como isto acontece:

a) colocaremos em $\sigma_{j}$ um sinal positivo com propabilidade

$\omega_{j}(+)=\frac{1}{2}\left(1+m_{j}\right)$

ou seja, quando

$\frac{1}{2}\left(1+m_{j}\right)>r^{\prime}, \operatorname{com} r^{\prime}$ sendo um número aleatório no intervalo entre 0 e 1 ;

b) colocaremos em $\sigma_{j}$ um sinal negativo com probabilidade

$\omega_{j}(-)=\frac{1}{2}\left(1-m_{j}\right)$, 
ou seja, quando $\frac{1}{2}\left(1+m_{j}\right)<r^{\prime}$.

c) de a) e b) resulta que $\sigma_{j}$ será +1 se $m_{j}>2 r^{\prime}-1$ e -1 se $m_{j}<2 r^{\prime}-1$. Por substituição dos valores extremos possíveis de $r^{\prime}(0 \mathrm{e} 1)$, vemos que está recuperada a prescrição em sua forma original, pois podemos obter $r($ entre $-1 \mathrm{e}+1)$ apartir de $r^{\prime}\left(r=2 r^{\prime}-1\right)$.

A confusão entre a prescrição NB e a do Modelo Cinético de Glauber (Glauber, 1963) deve ser evitada. Na dinâmica de Glauber, a probabilidade de invertermos o sinal de um spin $\sigma_{i}$ é dada por $W\left(\sigma_{i} \rightarrow-\sigma_{i}\right)=\frac{1}{2}\left\{1-\sigma_{i} \tanh \left[\beta J\left(\sum_{j} \sigma_{j}+h^{\prime}\right)\right]\right\}$, sendo $\sum_{j} \sigma_{j}$ o campo de troca realmente existente no momento de nos perguntarmos sobre a inversão do spin. Em NB, por outro lado, não se trata da inversão de spins, e sim da construção de um novo campo local num sítio, de forma que a verdadeira transição é a de um valor de $m_{i}$, no sítio $i$, para outro valor. Embora os $m_{j}$ sejam dados por tangentes hiperbólicas de somas de spin (como em Glauber), o campo no sítio $j$ não precisa ter relação instantânea com $m_{j}$. Além disso, sabemos que a prescrição de Glauber nos conduz à distribuição de Boltzmann, e não sabemos a qual distribuição nos leva o algoritmo de NB.

Vamos agora a uma cadeia de Ising. Dada a relação

$$
m_{i}=\tanh \left[\beta J\left(H_{i}+h^{\prime}\right)\right]=m\left(H_{i}\right),
$$

onde

$$
H_{i}=\sum_{j} \sigma_{j}
$$

com os valores de $\sigma_{i}$ dados pelo uso do algoritmo de NB nos sítios $j$, podemos iniciar a aplicação do algoritmo por uma certa configuração de spins, colocando em todos os sítios os valores correspondentes de $m_{i}$ ou, alternativamente, colocando em cada sítio valores quaisquer de $m\left(H_{i}\right)$, onde $H_{i}$ é uma variável que só pode possuir, $\operatorname{com} h^{\prime}=0$, os valores $0,2 \mathrm{e}-2$, em nosso caso linear. Vamos agora supor que focalizamos um sítio $i$ com um valor de $m_{i}$ a ser atualizado. São dados os valores de $m_{i+1}$ e $m_{i-1}$, ou seja, os valores das magnetizações locais nos vizinhos do sítio $i$. Após a iteração, $m_{i}$ passará ao valor $m^{\prime} i$ que pode ser igual a $m(2)=\tanh (2 \beta J), m(-2)=-m(2)$ e $m(0)=0$. As etapas para essa passagem são as seguintes:

a)em $i-1$ colocamos um spin +1 ou -1 com probabilidades

$$
\omega_{i-1}(+)=\frac{1}{2}\left(1+m_{i-1}\right) \quad \text { ou } \quad \omega_{i-1}(-)=\frac{1}{2}\left(1-m_{i-1}\right)
$$

respectivamente.

b) em $i+1$, colocamos os spins com probabilidades

$\omega_{i+1}(+)=\frac{1}{2}\left(1+m_{i+1}\right)$ ou $\quad \omega_{i+1}(-)=\frac{1}{2}\left(1-m_{i+1}\right)$

c) A probabilidade de $m_{i}$ (qualquer valor de $m\left(H_{i}\right)$ ) passar a $m(0)$ será constituída por duas possibilidades: ou no spin do sítio $i-1$ é colocado $\sigma_{i-1}=+1$ e no do sítio $i+1$,

$\sigma_{i+1}=-1$, ou no sítio $i-1$ colocamos $\sigma_{i-1}=-1$ e no sítio $i+1, \sigma_{i+1}=+1$. 
Assim temos

$W\left(m_{i} \rightarrow m^{\prime} i=m(0)\right)=\frac{1}{2}\left(1-m_{i-1} m_{i+1}\right)$

d) A probabilidade de $m_{i}$ passar a $m$ ( 2 ) é constituída pela possibilidade de que os spins dos sítios $i-l$ e $i+1$ passem a +1 . Teremos então

$W\left(m_{i} \rightarrow m_{i}^{\prime}=m(2)\right)=\frac{1}{4}\left(1+m_{i+1}\right)\left(1+m_{i-1}\right)$

e) A probabilidade de $m_{i}$ passar a $m$ ( - 2 ) é constituída pela possibidade de que os spins dos sítios $i-1$ e $i+1$ passem a -1. Dessa forma vem

$$
W\left(m_{i} \rightarrow m^{\prime} i=m(-2)\right)=\frac{1}{4}\left(1-m_{i+1}\right)\left(1-m_{i}-1\right) \text {. }
$$

As expressões acima mostram que podemos encarar a dinâmica do processo examinando a evolução dos valores de $m_{i}$. O processo é Markoviano, pois as probabilidades de transição dependem apenas do estado anterior. Apesar disso, não é simples o exame da evolução estocástica, de maneira que não sabemos qual deve ser a distribuição estacionária de probabilidades para as configurações $\left\{m_{i}\right\}$. Entretanto, não será nosso objetivo estudar aqui essa dinâmica. Enfatizamos ainda que o campo de troca local, por exemplo, no sítio $i$ - 1, só é alterado quando este sítio estiver sendo focalizado, pois quando estivermos, por exemplo, no sítio $i+1$, vamos alterar o spin do sítio $i$, mas apenas para reconstruir o campo em $i+1$, o que não muda o campo de $i-1$. Vamos agora à interpretação dos autores sobre $o$ algoritmo.

Segundo seus autores esse algoritmo seria uma das formas de obter soluções de um conjunto de equações de consistência, com os $m_{i}$ redefinidos como $\left.m_{i} \equiv<\sigma_{i}\right\rangle$, na forma

$$
<\sigma_{i}>=m_{i}=\sum_{\left\{\sigma_{j}\right\}} \prod_{j}\left(\frac{1+\sigma_{j} m_{j}}{2}\right) \tanh \left[\beta J\left(\sum_{j} \sigma_{j}+h^{\prime}\right)\right],
$$

com $j$ indicando os primeiros vizinhos do sítio $i$ e com $\left\{\sigma_{j}\right\}$ representando um conjunto de valores possíveis para os spin $\sigma_{j}$. Estas equações representariam os cálculos de $\left\langle\tanh \left[\beta J\left(\sum_{j} \sigma_{j}+h^{\prime}\right)\right]\right\rangle$ sobre uma distribuição fatorizada para uma configuração $\{\sigma\}$ de spins na rede na forma

$$
P\{\sigma\}=\prod_{i} \frac{1}{2}\left(1+\sigma_{i} m_{i}\right) .
$$

Ainda alternativamente, conforme Banavar, Cieplak e Maritan (Banavar,1991), esse sistema de equações pode ser obtido, a campo nulo, por meio de expansões em produtos dos spins $\sigma_{j}$ para $\tanh \left[\beta J\left(\sum_{j} \sigma_{j}\right)\right]$ aplicando-se então uma fatorização para as médias desses produtos. Estas afirmações estão mostradas com um pouco mais de detalhe no Apêndice 2, onde discutimos também as equações de consistência para uma rede ferromagnética. Para os casos de redes ferromagnéticas ou antiferromagnéticas quadradas, este esquema conduz a soluções relativamente não muito complicadas sob imposição de homogeneidade por subrede. Em geral, segundo os autores do algoritmo, poderia haver muitas soluções, bastante difíceis de obter, principalmente para casos de modelos frustados, onde se cogita que a imposição de homogeneidade por subredes poderia levar a soluções não muito interessantes, ou de menor relevância, tornando a implementação estocástica das equações particularmente útil (Netz, 1991 b e c). 
Os casos primeiramente analizados pelos autores foram o de uma rede antiferromagnética plana triangular e o de uma rede espacial, superposição destas últimas e com interações também antiferromagnéticas entre esses planos. As vantagens da aplicação de seu método, na opinião dos autores, estariam, como já citado, no fato de se poder distinguir efeitos como o de dimensão da rede e da possibilidade de se obter bons resultados numéricos com amostragens substancialmente menores do que as necessárias nos tratamentos convencionais de Monte Carlo. Essas últimas afirmações são feitas, porém, sem nenhuma análise de erros.

A fim de procurarmos a correspondência entre o algoritmo e a imagem que dele fazem os autores, fizemos testes em redes quadradas ferromagnéticas, que são muito conhecidas. Os resultados que obtivemos estão no capítulo seguinte.

Desejamos finalizar este capítulo apresentando um caminho heurístico para a obtenção da prescrição NB. Lembrando um pouco a seção anterior, vimos que a hamiltoniana de teste $\mathrm{H}_{\mathrm{o}}$ para o modelo de Ising era dado por

$$
H_{0}=-J \sum_{i} H_{i} \sigma_{i}-h \sum_{i} \sigma_{i},
$$

levando à função de partição

$$
Z_{0}=\prod_{i} 2 \cosh \left[\beta J\left(H_{l}+h^{\prime}\right)\right] \quad\left(h=J h^{\prime}\right) .
$$

Dessa forma, a distribuição de probabilidades para $H_{\circ}$ pode ser escrita como

$$
\begin{aligned}
P_{o}\{\sigma\} & =\frac{e^{-\beta H_{0}}}{Z_{o}} \\
& =\prod_{i} \frac{e^{\beta J\left(H_{i}+h^{\prime}\right) \sigma_{i}}}{2 \cosh \left[\beta J\left(H_{i}+h^{\prime}\right)\right]} \\
& =\prod_{i} \frac{e^{\beta J\left(H_{i}+h^{\prime}\right) \sigma_{i}}}{2 \cosh \left[\beta J\left(H_{i}+h^{\prime}\right) \sigma_{i}\right]} .
\end{aligned}
$$

Então temos

$$
\begin{aligned}
\boldsymbol{P o}_{\boldsymbol{o}}\{\boldsymbol{\sigma}\} & =\prod_{i} \frac{1}{2}\left\{1+\tanh \left[\beta J\left(H_{i}+h^{\prime}\right) \sigma_{i}\right]\right\} \\
& =\prod_{i} \frac{1}{2}\left\{1+\sigma_{i} \tanh \left[\beta J\left(H_{i}+h^{\prime}\right)\right]\right\} .
\end{aligned}
$$

A distribuição acima é fatorizada, sendo a distribuição para um spin $\sigma_{j}$ dada por

$$
P_{o_{j}}\left(\sigma_{j}\right)=\frac{1}{2}\left\{1+\sigma_{j} \tanh \left[\beta J\left(H_{j}+h^{\prime}\right)\right]\right\} .
$$

Vemos que a forma acima é idêntica à da probabilidade prescritiva

$$
\omega_{j}\left(\sigma_{j}\right)=\frac{1}{2}\left(1+\sigma_{j} m_{j}\right),
$$

usada por NB para a reconstrução da magnetização local no sítio $i$, vizinho a $j$, com a diferença de que em NB os valores de $m_{j}$ são sempre dados por 


$$
m_{j}=\tanh \left[\beta J\left(\sum_{l} \sigma_{l}+h^{\prime}\right)\right],
$$

com $l$ representando os vizinhos de $j$ e com a soma representando o campo local no sítio $j$ na última iteração sobre esse sítio.

Temos então uma receita para obter a prescrição NB para outros modelos. Procuramos a distribuição da hamiltoniana de teste $H_{0}$, cuja forma será idêntica à da prescrição procurada, deixando-se apenas que os $H_{i}$ sejam campos locais " reais" numa certa iteração, e não campos "efetivos", como os dados na equação de consistência, para o modelo em questão. 


\section{III - A Aplicação do Algoritmo Sobre a Rede Quadrada Ferromagnética de Ising}

\section{III.1 - O desenvolvimento do algoritmo}

Sintetizando a descrição que já fizemos anteriormente, podemos dizer que as etapas para a atualização de uma "magnetização local" $m_{i}$ são as seguintes:

a) localização dos sítios $j$ primeiros vizinhos de $i$, colocando em cada $j$ um spin de sinal

$m_{j}-r$, com $r$ sendo um número aleatório entre -1 e +1 . Sinteticamente, fazemos

$\sigma_{j}=\operatorname{sinal~de}\left(m_{j}-r\right) ;$

b) atualização de $m_{i}$ fazendo-se

$$
m_{i}=\tanh \left[\beta J\left(\sum_{j} \sigma_{j}+h^{\prime}\right)\right] .
$$

Vamos ver agora como realizamos a aplicação do algoritmo NB para a rede quadrada ferromagnética a campo nulo. Escolhemos sempre partir de uma configuração $\left\{m_{i}\right\}$ inicial. Observemos que os valores possíveis de $m_{i}$ serão, sempre, $\tanh (4 / T),-\tanh (4 / T), \tanh (2 / T)$,

$-\tanh (2 / T)$ e $0, \operatorname{com} T \equiv \frac{1}{\beta J}$. Fazemos então uma tabela com os possíveis valores de $m_{i}$ para um dado $T$. Estivemos sempre trabalhando com uma versão sequencial, na qual percorremos a rede sítio a sítio aplicando a prescrição de atualização da magnetização local. Cada corrida completa sobre a rede é um passo de Monte Carlo (PMC). Para cada PMC guardamos o valor de $\left(\Sigma_{i} m_{i}\right) /(\mathrm{N} \times \mathrm{N})$, para uma rede de $\mathrm{N}$ x N spins, ou de alguma outra grandeza que possa ser considerada importante. Dado então um certo número de PMC, descontados alguns passos iniciais, calculamos a média dos valores acumulados.

Uma observação importante diz respeito ao fato de Netz e Berker não utilizarem, na definição da magnetização, os valores absolutos guardados em cada passo de Monte Carlo. Pelo contrário, acumulam, como afirmamos no parágrafo acima, as soma dos spins junto a seus valores relativos em cada passo*. Como mostraremos mais tarde, essa grandeza revelou-se aparentemente inviável para a obtenção de resultados numéricos confiáveis (ao menos no caso dos modelos analizados neste trabalho), obrigando-nos ao uso de uma outra grandeza para esse fim (escolhemos a energia por sítio, como veremos).

Damos, no Apêndice 4, a forma geral do algoritmo para a rede ferromagnética a um dado valor de $T$, que foi desenvolvida por Luís Femandez Lopez e por nós.

* Isso deduz-se das curvas de magnetização de subrede em função da temperatura a campo nulo apresentadas pelos autores (Netz, $1991 \mathrm{a}, \mathrm{b}, \mathrm{e} \mathrm{c}$ ) onde observamos trocas de posições dos caracteres representativos de cada subrede ( três, em seus modelos) ao longo da temperatura, o que só é possível para a magnetização como definida acima. 


\section{III.2 -Resultados Qualitativos para a Magnetização por spin}

Nāo fizemos um trabalho numérico confiável para a magnetização. Isso porque, em primeiro lugar, com já dissemos e mostraremos abaixo, a grandeza sugerida pelos autores do algoritmo para representar a magnetização a campo nulo não permite a apresentação de resultados bons. Além disso, seguindo a sugestão de D.P. Landau (Landau, 1976) usamos o ponto de inflexão na curva da energia por spin em função da temperatura para representar a "temperatura de Curie" das redes com que trabalhamos, razão pela qula optamos por fazer uma análise mais aprofundada da energia. Apresentaremos aqui, porém, alguns resultados para a magnetização por spin que nos ajudam a fazer algumas observações iniciais sobre o método.

Observemos então as curvas abaixo (Fig. 01), que representam $M=\mid<\Sigma m_{i} /(N \times N)>1$ em função da temperatura $T$ (em unidades de $\beta J$ ) a campo nulo para as redes $4 \times 4$ (indicada por losangos), $10 \times 10$ (indicada por triângulos) e $60 \times 60$ (indicada por círculos).

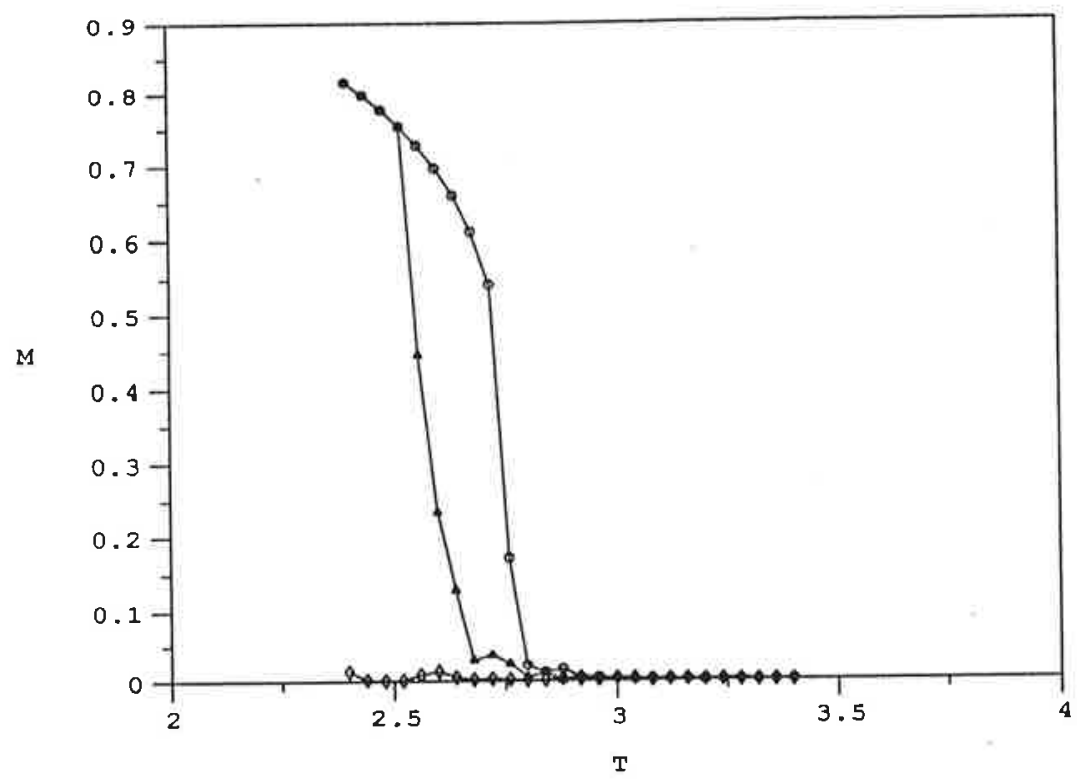

Fig.01

Esses gráficos, de forma muito irregular, são obtidos com o mesmo número de passos de Monte Carlo usados para o cálculo da energia em função da temperatura $\left(10^{6}\right.$ para rede 4 x $4,6.5 \times$ $10^{4}$ para rede $10 \times 10$ e $1.25 \times 10^{4}$ para rede $60 \times 60$ ).

Lembrando que o número máximo de PMC usados pelos autores do algoritmo é 500 para redes de 24 × 24 × 6 spins (Netz, 1991 a e c) observemos aqui a dificuldade de obtenção de curvas suaves para $M \times T$. O processo que ocorre aqui é semelhante ao que acontece nos algoritmos conhecidos de Monte Carlo onde, dada a finitude do sistema, a migração entre estados de magnetização inversa pode ocorrer muito frequentemente, mesmo a temperaturas baixas. Normalmente, para contornar o problema, sugere-se a acumulação de valores absolutos das magnetizações em cada $\mathrm{PMC}$, de forma que a baixas temperaturas o sistema, que sempre fica muito pouco tempo em estados de magnetizações pequenas ou nulas, é considerado em sua fase ferromagnética, não obstante oscilações entre valores extremos e de sianis opostos de magnetização (Binder, 1988). Pode-se demomstrar que no limite termodinâmico e com campo externo tendendo a 0 temos $\left\langle\left|\Sigma \sigma_{i}\right|\right\rangle \rightarrow<$ $\Sigma \sigma_{i}>$, o que torna cabível a redefinição da magnetização em simulações da distribuição de Gibbs (Binder, 1988). 
Uma questão que já cabe discutir nessa seção diz respeito ao fato de os autores sugerirem que o método gera soluções de auto-consistência. De fato, se entendermos essa expressão como significando

$$
<\frac{1}{N^{2}} \sum_{i} \sigma_{i}>=<\frac{1}{N^{2}} \sum_{i} m_{i}>,
$$

com os $m_{i}$ entendidos como já explicamos e com $<\ldots>$ representando

$$
\frac{1}{N \text { ode } P M C} \sum_{\text {todos os } P M C} \ldots,
$$

não podemos afirmar que não haja alguma auto-consistência qualitativa, apesar de observarmos que quase sempre ocorreram pequenas diferenças, imperceptíveis na escala dos gráficos acima (Fig 01), em geral no sentido de fazer

$$
<\frac{1}{N^{2}} \sum_{i} \sigma_{i}>><\frac{1}{N^{2}} \sum_{i} m_{i}>.
$$

Entretanto, já dissemos que essas grandezas, aparentemente, não servem para a análise numérica. Observemos agora os gráficos de $\left\langle\left|\frac{1}{N^{2}} \sum_{i} \sigma_{i}\right|\right\rangle \quad$ (indicados por triângulos) e $<\left|\frac{1}{N^{2}} \sum_{i} m_{i}\right|>\quad$ (indicados por círculos) para as redes $4 \times 4$ e $10 \times 10$ em função de $T$ ( Figs. $02 \mathrm{e}$ 03).

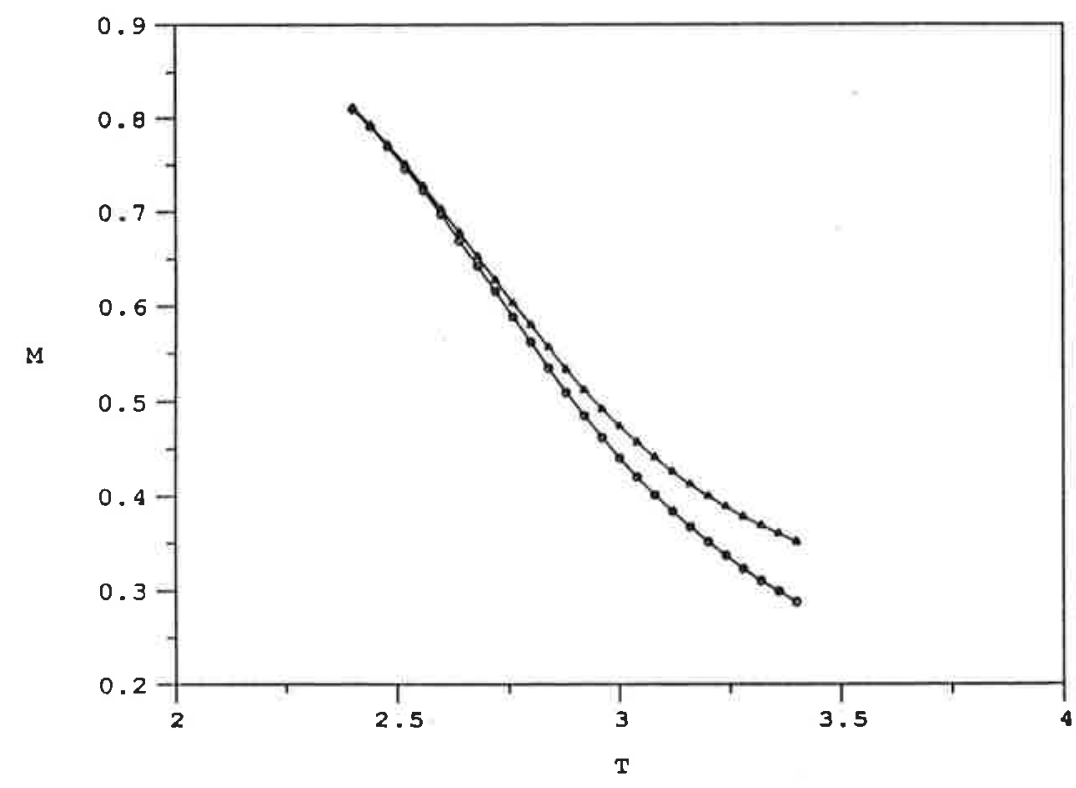




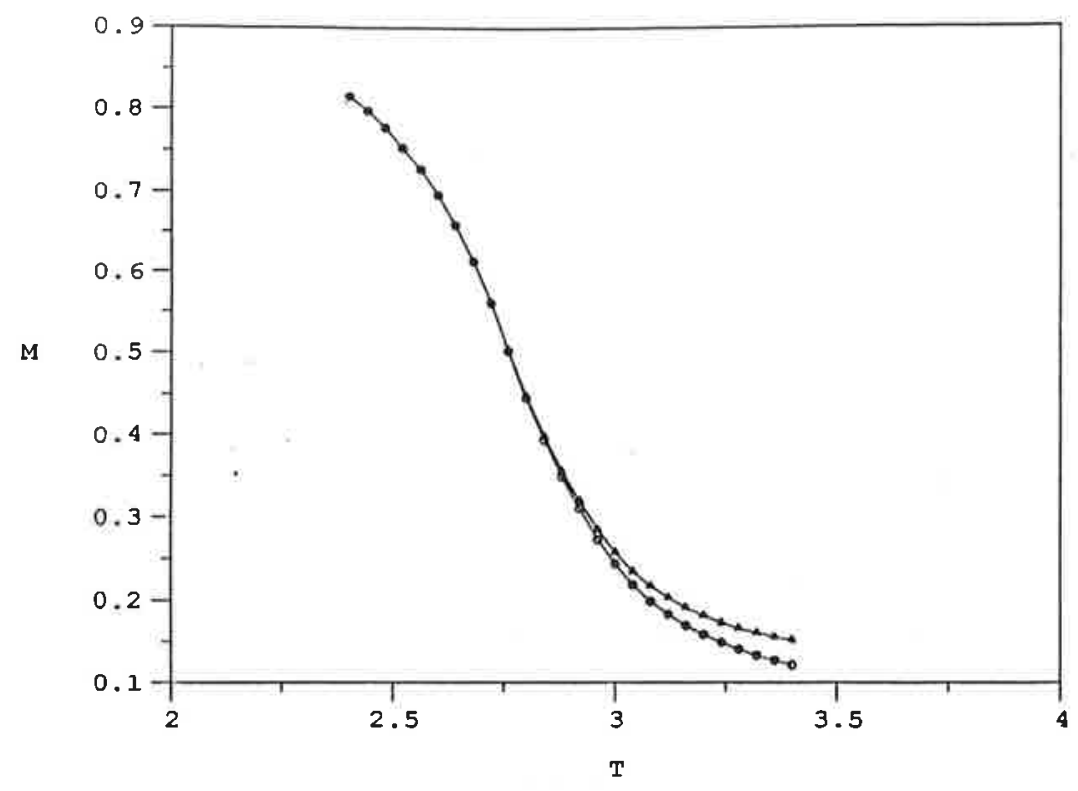

Fig.03

Para o levantamento das curvas acima utilizamos as mesmas amostragens já indicadas, usadas nos cálculos de energia que mostraremos. Não vemos, aqui, nada parecido com uma consistência, no sentido acima apontado, e ficamos com uma certa dificuldade em perceber com clareza, ou seja, pelo uso de uma grandeza que sirva para descrições numéricas, em que sentido poderíamos falar em auto-consistência.

Como observação final, podemos dizer que as últimas duas curvas sugeram a existência de um efeito de escala ("finite-size scaling"), onde deve haver uma convergência de comportamento com $\mathrm{N}$ crescente. Em seção posterior apresentaremos os resultados para a energia por spin para as erdes $4 \times 4,10 \times 10$ e $60 \times 60$, mostrando o efeito de escala gerado por esse algoritmo no cálculo dessa grandeza, e uma extrapolação da temperatura de Curie para $\mathrm{N} \rightarrow \infty$. Anteriormente, porém, daremos explicações sobre o método de análise de erros usado. 


\section{III-3. Análise de Erros pelo Método de Ineficiência Estatística}

Uma tendência geral de simulaçōes de Monte Carlo do tipo "amostragens por importância" se revelou nas simulações com o algoritmo NB: a existência de correlações temporais fortes entre configurações sucessivas (passos de Monte Carlo). Uma vez que não podemos considerar cada um dos valores de uma sequência de dados tirados do processo como estatisticamente independentes , a análise dos erros se complica. Assim, mesmo quando a carta de controle do processo, que é o gráfico do valor de uma grandeza em questão em função do tempo ( passos de Monte Carlo percorridos), indica a estabilização em torno do valor médio conhecido para aquelas condições ( em nosso caso a temperatura), não podemos usar a análise de erros "standard", que parte do pressuposto de independência estatística.

O tratamento modernamente mais aceito para a análise de erros em simulações de Monte Carlo se baseia no cálculo das correlações temporais entre pares de dados obtidos no processo (Binder, 1988). Entretanto, usamos neste trabalho um método mais antigo, proposto por Friedberg e Cameron (Friedberg, 1970) e usado por D.P. Landau em seu tratamento do problema dos efeitos de tamanho finito para o modelo de Ising em redes quadradas ( Landau, 1976), chamado de Análise de Ineficiência Estatística. Embora talvez menos intuitivo do que o tratamento por correlações temporais, esse método é, aparentemente (segundo D.P. Landau), mais simples de ser implementado. Apresentamos no Apêndice 3 uma prova, devida a Vera B. Henriques, da equivalência entre as análises de Ineficiência Estatística e de correlações temporais.

O método consiste em dividirmos uma sequência de $M$ dados obtidos em uma corrida Monte Carlo em blocos de tamanho $P$. Destes $M$ passos já descontamos uma certa quantidade de passos iniciais. Haverá $M P$ blocos, de forma que $M=P M P$. A média em um bloco $i$ é dada por

$$
\bar{X}_{i}=\frac{1}{P} \sum_{j=1}^{P} X_{j}^{i},
$$

onde o índice $j$ representa o contador dos $p$ valores da grandeza $X$, com $i$ referindo-se sempre ao $i$ ésimo bloco em consideraçāo. O valor esperado da grandeza, $\bar{X}$, que é a média para todos os valores da sequência, será

$$
\begin{aligned}
\bar{X} & =\frac{1}{M_{P}} \sum_{i=1}^{M_{P}} \overline{X_{i}} \\
& =\frac{\text { soma total dos valores sucessivos de } X}{M} .
\end{aligned}
$$

A variância total dos dados é

$$
\sigma^{2}=\frac{1}{M} \sum_{i=1}^{M_{P}} \sum_{j=1}^{P}\left(X_{j}^{i}-\bar{X}\right)^{2}
$$

e a variância das $M P$ médias

$$
\sigma_{P}^{2}=\frac{1}{M_{P}} \sum_{i=1}^{M_{P}}\left(\overline{X_{i}}-\bar{X}\right)^{2}
$$


O que acontece, na medida em que partimos de um valor baixo de $P$ ( $\operatorname{para} P=1$, $\sigma_{P}^{2}$ se reduz a $\sigma^{2}$ ) é que o valor de

$$
\varepsilon_{p}^{2}=\frac{\sigma \mathscr{P}}{M P}
$$

tende a aumentar sempre $\operatorname{com} P$ até se estabilizar para um determinado limite. $O$ mesmo acontece com a grandeza

$$
I(P)=\frac{\varepsilon^{2}}{\frac{\sigma^{2}}{M}}=\frac{M \varepsilon^{2}}{\sigma^{2}} .
$$

Esse comportamento geral de $\varepsilon^{2} \operatorname{P}$ e $I(P)$ tem explicação dada no Apêndice 3. Vamos observar abaixo ( Fig.4) uma tabela característica de $\mathcal{E} P$ como função de $P$, estando em questão a energia por spin de uma rede $4 \times 4$, e sendo a temperatura $T=2.4$ ( próxima da de "transição" para essa rede) numa simulação com o algoritmo NB.

\begin{tabular}{|c|c|c|}
\hline$P$ & $\varepsilon P\left(\times 10^{3}\right)$ & Erro em $\varepsilon P\left(\delta P \times 10^{3}\right)$ \\
\hline 1 & 4.697 & 0.03 \\
\hline 10 & 6.954 & 0.14 \\
\hline 20 & 7.493 & 0.21 \\
\hline 25 & 7.692 & 0.25 \\
\hline 50 & 7.895 & 0.35 \\
\hline 100 & 8.098 & 0.52 \\
\hline 250 & 8.888 & 0.90 \\
\hline 500 & 7.913 & 1.10 \\
\hline 1250 & 8.404 & 2.20 \\
\hline 2500 & 6.415 & 2.30 \\
\hline
\end{tabular}

Fig .04

Podemos observar que $\varepsilon P$ não se estabiliza muito suavemente. Porém, é possível uma avaliação, dada uma amostragem de $M$ passos, desse valor $\varepsilon$ de estabilização, tomando $\varepsilon$ como o maior valor de $\varepsilon P$ antes de sua primeira queda com $P$. Assim, com os dados da Fig. 4, elegemos, $\varepsilon=(8.9 \pm 0.9) \times 10^{-3}$ correspondendo a $P=250$.

Como a própria definição de $\varepsilon^{2} P$ sugere, $\varepsilon$ será considerado o erro na medida, isto é, consideraremos os $M P$ valores de $\overline{X_{i}}$, para o $P=P$ máx onde consideramos estabilizado o valor de $\varepsilon P$, como dados estatisticamente independentes. Então

$$
\varepsilon=\frac{\sigma P_{\max }}{\sqrt{M P_{m a x}}}
$$

As incertezas mostradas na Fig. 4 correspondem a avaliação dos erros em $\varepsilon P$, denotados por $\delta P$, e calculados por

$$
\delta P=\frac{\varepsilon P}{\sqrt{2\left(M_{p}-1\right)}}
$$


Essa relação só deve ser estritamente válida quando $\varepsilon P$ se estabiliza. Dessa forma, a incerteza no erro estatístico será avaliada pela relação acima $\operatorname{com} \varepsilon P=\varepsilon$ e $M P=M P_{\max }$.

Ao valor de $I\left(P=P_{m a ́ x}\right)$ chamamos inefíciência estatística, denotado por $I$, que será

$$
I=I\left(P=P_{\text {máx }}\right)=\frac{M\left(\varepsilon_{P_{\text {max }}}\right)^{2}}{\sigma_{2}}=\frac{M \varepsilon^{2}}{\sigma^{2}} .
$$

A ineficiênçia estatística possui duas interpretações. Consideremos primeiramente, a fór-

mula

$$
\left(\varepsilon_{P=1}\right)^{2}=\frac{\sigma^{2}}{M},
$$

que nos daria o erro na medida caso os $M$ dados fossem descorrelacionados. Então teremos

$$
\varepsilon^{2}=(I)\left(\varepsilon_{1}\right)^{2},
$$

e a ineficiência estatística pode ser interpretada como sendo um "fator de ampliação" do erro estatístico devido às correlações. Dessa forma, se temos uma idéia, em nosso caso, de como $I$ varia com a temperatura, podemos ajustar uma curva $I x T$ e calcular os erros dentro de uma certa margem de confiança. Outra interpretação para $I$ pode ser dada da seguinte forma: para obtermos um erro igual a $\varepsilon_{1}$ em nossa medida, precisaremos aumentar nossa estatística, isto é, aumentar o valor de $M$ até um valor $M^{\prime}$, de forma que

$$
I=\frac{M^{\prime} \varepsilon^{2}}{\sigma^{2}}=\frac{M^{\prime}}{\sigma^{2}}\left(\frac{\sigma^{2}}{M}\right)=\frac{M^{\prime}}{M},
$$

e a ineficiência estatística avalia o quanto uma amostragem $M$ deveria ser maior para que obtivéssemos um erro equivalente a $\varepsilon_{1}$, calculado pela amostragem original.

Considerando novamente os dados da Fig.4, isto é, dados $\varepsilon$, $\varepsilon$, e a avaliação do erro de $\varepsilon$ que vêm dos valores da tabela, calculamos a ineficiência estatística naquelas circunstâncias por $I=(3.6 \pm 0.7)$. 
III.4 - Apresentação de resultados a campo nulo com base em cálculos de energia por spin

\section{1) Cartas de controle}

Vamos observar algumas cartas de controle da energia, em cada PMC, versus o número de passos de Monte Carlo. Primeiramente, temos abaixo (Fig.5) a evolução da energia para uma rede $60 \times 60$ a uma temperatura $T=4.0$, que sabemos por inspeção ser bem maior que a de "transição" para essa rede, e onde partimos de uma configuração desfavorecida, com todos os valores de $m_{i}$ iguais a 1.0.

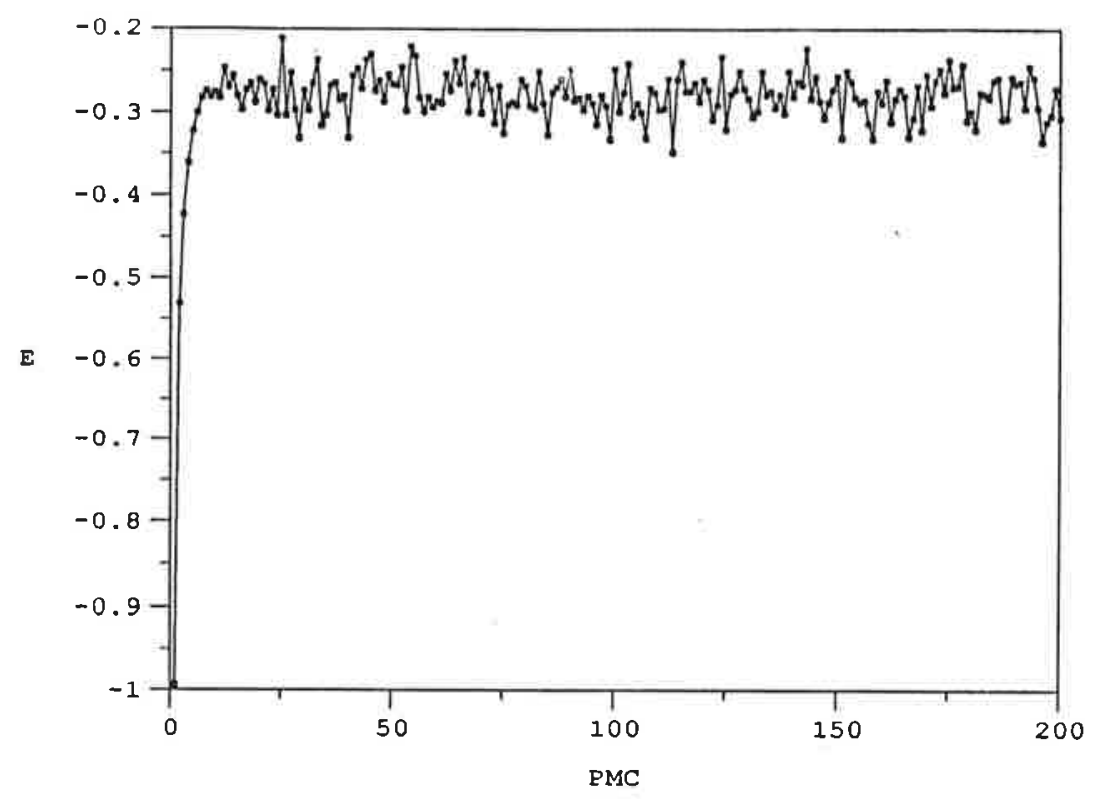

Fig.05

O gráfico acima representa uma tendência muito geral de rápida estabilização da energia em torno de um valor médio quando estamos fora da região de transição, fato praticamente independente do tamanho da rede e também da inicialização pois, como dissemos, começamos por uma configuração francamente desfavorecida dos valores de $m_{i}$. Esse comportamento, entretanto, não ocorre quando estamos perto do ponto de transição da rede. Em sequência, temos as cartas para $\mathrm{N}=10$ e $\mathrm{N}=60 \operatorname{com} T=2.72$ ( Figs. 6 e 7 ).

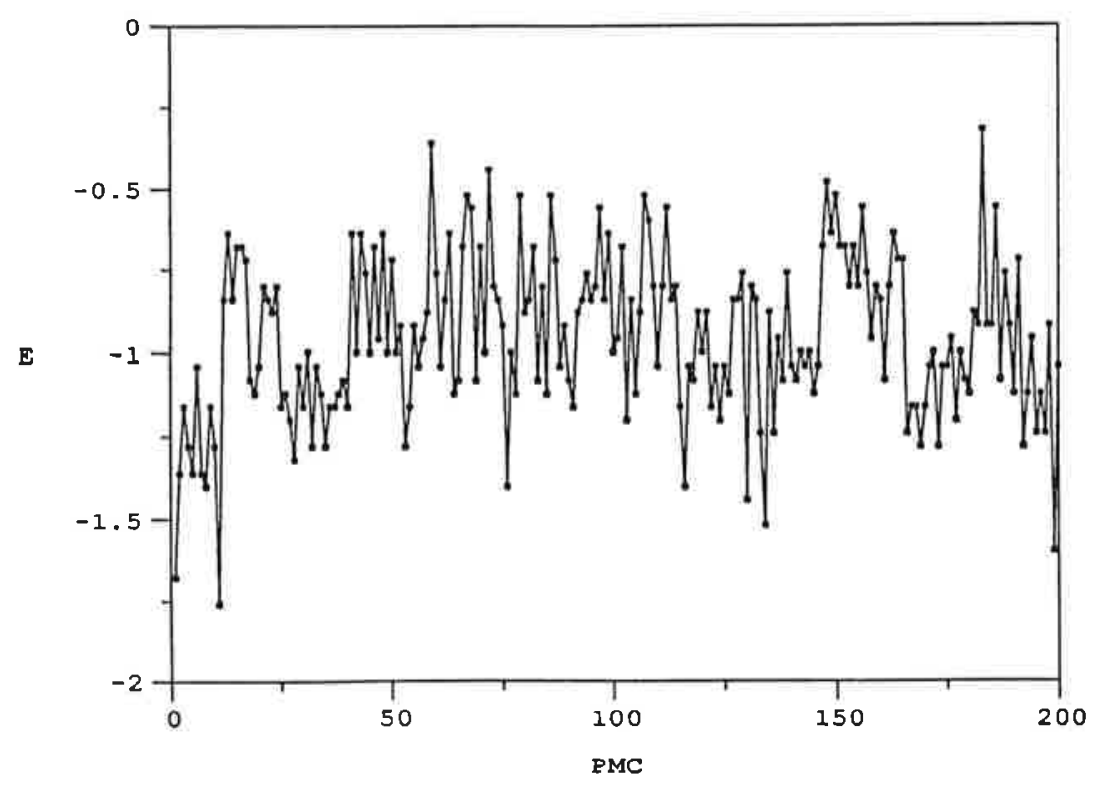




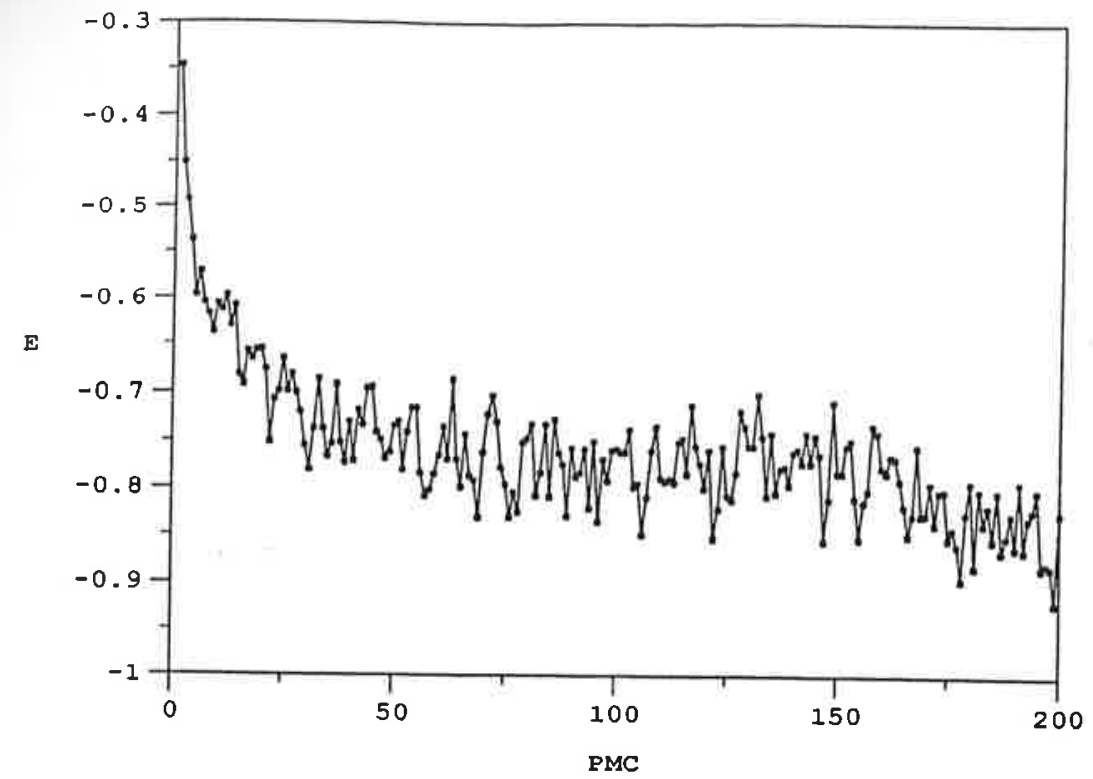

Fig.07

Percebemos das curvas acima que a estabilização se torna mais lenta perto do ponto de $\mathrm{Cu}$ rie na medida em que aumentamos o tamanho da rede. Esse fato também caracteriza as simulações da distribuição de Gibbs ( Binder, 1988 ). Para minimizar esse fato é aconselhável tomar como configuração inicial, para uma certa temperatura, uma configuração $\left\{m_{i}\right\}$ de equilíbrio gerada em uma outra temperatura próxima. Assim, por exemplo, numa corrida para várias temperaturas, podemos começar de $T$ baixo partindo da configuração com todos os $m_{i}$ iguais a 1, inicializando apenas essa primeira vez, de maneira que para um dado valor de temperatura começaremos pela última configuração gerada na temperatura anterior. Em todas as nossas simulações fizemos o descarte dos primeiros 50 PMCs, o que, por comparação em rodadas independentes, se mostrou satisfatório.

2) Ineficiência Estatística

O gráfico abaixo (Fig.08) representa alguns valores de ineficiência estatística para as redes $4 \times 4$ e $10 \times 10$ em função da temperatura. A rede $4 \times 4$ está indicada por triângulos e a $10 \times 10$ por círculos.

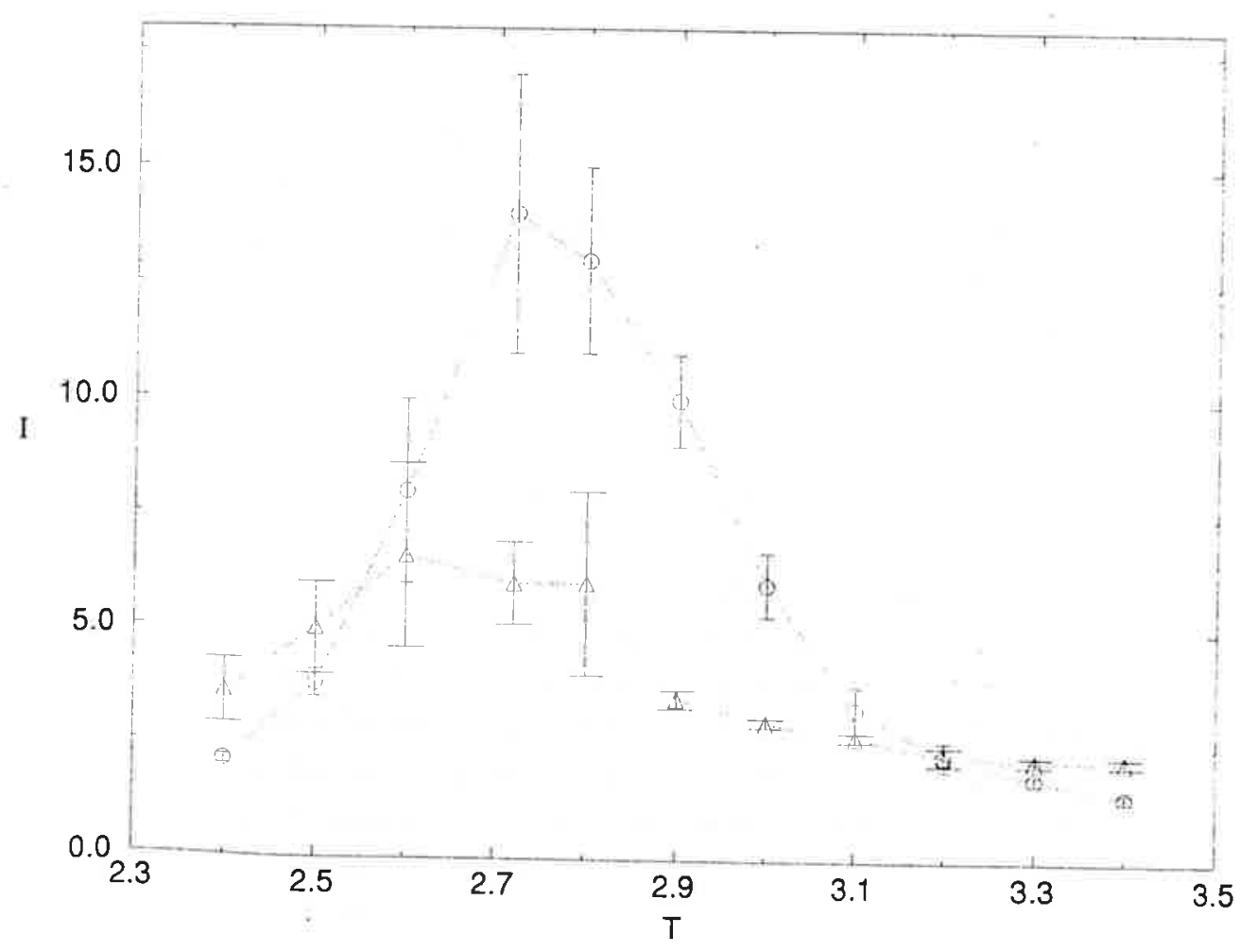


Dada a interpretação da ineficiência estatística, observamos pelos gráficos acima uma tendência ao aumento das correlações temporais nas proximidades do ponto de transição ao aumentarmos o tamanho da rede. Nossos resultados para a rede de $60 \times 60$ sítios, embora talvez menos confiáveis, indicam uma grande elevação, em relação às de 4 x 4 e $10 \times 10$, nos valores da ineficiência estatística nas proximidades do ponto de transição (ponto de inflexão da curva de energia $x$ temperatura).

Temos, a essa altura, condições de compararmos o número de passos de Monte Carlo necessários para a determinação de um valor de energia, pelo algoritmo NB, com o necessário em simulações da distribuição de Gibbs nas proximidades da temperatura de transição. Tomaremos como base o trabalho de D.P. Landau, onde se impõe, nas proximidades de Tc, um erro na ordem de $1 \%$ na energia interna (Landau, 1976). Na Figura 09 mostramos uma tabela em que todas as colunas, com excessão da última, são tiradas de cálculos usando-se o algoritmo NB. Na tabela, N é o número de sítios ao longo de uma direção ( a rede é $\mathrm{N} \mathrm{x} \mathrm{N}$ ), $E$ é a energia por sítio nas proximidades da temperatura de transição, $\sigma^{2}$ a variância dos valores de $E$ (como definida na seção anterior) e $I$ a ineficiência estatística correspondente (lembramos que $I=M \times \frac{(\text { erro })^{2}}{\sigma^{2}}$ ).

\begin{tabular}{|c|c|c|c|c|c|}
\hline$N$ & $E$ & $\sigma^{2}$ & $I$ & $\begin{array}{c}\text { PMC } \\
\text { Necessários em } \\
\text { NB } \\
\text { (para erro de 1\% } \\
\text { em } E \text { ) }\end{array}$ & $\begin{array}{c}\text { Amostragem } \\
\text { necessária em } \\
\text { D.P. Landau }\end{array}$ \\
\hline 4 & $-1.12(T=2.60)$ & 0.34 & $7 \pm 2$ & $13000-24000$ & $20000-50000$ \\
\hline 10 & $-0.901(T=2.72)$ & $7.6 \times 10^{-2}$ & $14 \pm 3$ & $10000-15000$ & $4000-10000$ \\
\hline 60 & $-0.895(T=2.72)$ & $2.43 \times 10^{-3}$ & da ordem de 400 & da ordem de 12000 & $1000-2500$ \\
\hline
\end{tabular}

Os dados da tabela acima não parecem favorecer o algoritmo de NB para as redes quadradas ferromagnéticas no que se refere a cálculos de energia. Pelo contrário, na medida em que aumentamos o tamanho das redes, parece haver a tendência à necessidade de uma amostragem muito maior em NB do que em Metrópolis ( algoritmo usado por D.P. Landau). Além disso, como explicaremos no ponto seguinte, julgamos necessária a utilização de amostragens substancialmente maiores, para a determinação dos valores de $E$, devido à forma com que avaliamos o calor específico por spin.

\section{3)Energia Interna e Calor Específico em função da temperatura}

As curvas da Figura 10 mostram o comportamento de energia em função da temperatura para as três redes utilizadas. A rede $4 \times 4$ está indicada por losangos, a $10 \times 10$ por triângulos e a 60 x 60 por círculos. Sempre nos limitamos a uma região de temperaturas próximas da transição. As amostragens utilizadas foram: $10^{6}$ para $\mathrm{N}=4,65000$ para $\mathrm{N}=10$ e 12500 para $\mathrm{N}=60$. A necessidade do uso dessas amostragens tão grandes está no fato de termos utilizado os dados das curvas energia $\mathrm{x}$ temperatura para esboçar as curvas de calor específico avaliando as derivadas da energia em cada temperatura. Uma vez que a avaliação de derivadas introduz erros consideráveis, tivemos de aumentar muito as amostragens. 


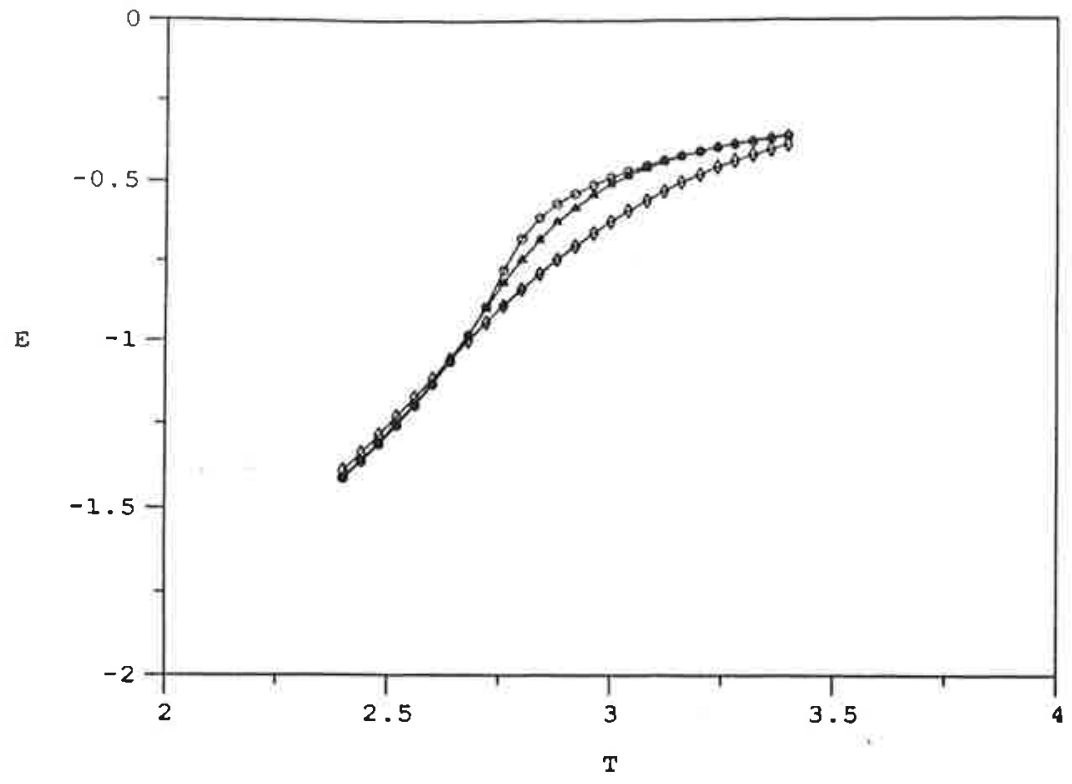

Fig.10

A Fig. 11 mostra as curvas de calor específico por spin, em unidades de $K_{B}$, em função da temperatura. A avaliação do calor específico em uma temperatura $T_{n}, \operatorname{com} n$ representando uma das 26 temperaturas nas curvas $E \times T$ (Fig.11), se fez pela fórmula $C_{n}=\frac{E\left(T_{n+1}\right)-E\left(T_{n}\right)}{T_{n+1}-T_{n}}$, sobre a qual aplicamos a propagação de erros nos pontos de calor específico máximo, considerando as incertezas no cálculo de energia. Assim, os valores máximos do calor específico são da ordem de $1.45 \pm 0.05$, para a rede $4 \times 4,2.1 \pm 0.1$ para a rede $10 \times 10$ e cerca de $2.9 \pm 0.3$ para a rede $60 \times 60$. A linha pontilhada na Fig. 11 representa o calor específico na aproximação de Bethe-Peierls ( Pathria, 1972 ), cuja temperatura de Curie, de 2.88..., é a que está mais próxima da de NB entre as formas aproximativas existentes do problema. Na figura 11, os losangos representam a rede $4 \times 4$, os triângulos a $10 \times 10$ e os círculos a $60 \times 60$.

Um fato que notamos no caso do algoritmo NB é que há uma total discordância entre o calor específico avaliado como fizemos o calculado por flutuações na energia por spin. Estas flutuações são dadas pelo valor de $\sigma^{2}$, e o calor específico calculado por elas seria $C_{f}=\frac{\mathrm{N}^{2} \sigma^{2}}{T^{2}} *$. Mas, se observarmos os valores de $\sigma^{2}$ (Fig. 09) para as redes usadas, nas temperaturas de transição, teríamos, para $C_{f}$, algo da ordem de 0.8 para a rede $4 \times 4(\operatorname{com} T c=2.6)$ e de 1.0 para as redes 10 x10 e $60 \times 60$ (com $T c=2.72$ ), valores esses que estão em confronto com os dados mais acima. Esse fato, que é o reflexo de uma tendência geral de redução das flutuações no ponto de transição com o aumento de $\mathrm{N}$, impede o uso dessas flutuações para a definição do calor específico, e pode ser interpretado como um reflexo do fato de não estarmos lidando com uma simulação da distribuição de Gibbs.

* Netz e Berker calculam assim curvas de calor específico em seu primeiro artigo ( Netz, 1991 a ), curvas essas que não constam de seu 20 artigo ( Netz, 1991 c). 


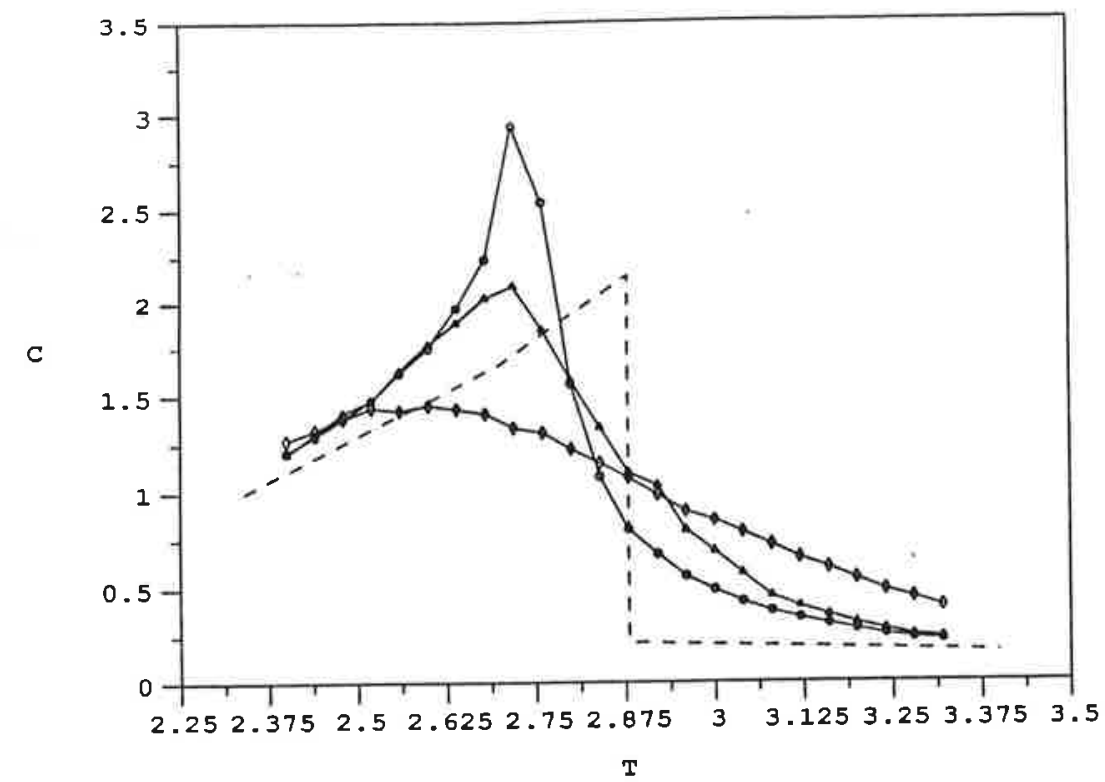

Flg.11

4) Extrapolação para $N \rightarrow \infty$

A curva seguinte ( Fig.12) representa a variação, com N ${ }^{-1}$, da temperatura "de Curie" correspondente ao máximo do calor específico. Fizemos simulações de mais algumas redes, além das discutidas, para uma melhor obtenção da forma da curva $T_{c} \times \mathrm{N}^{-1}$, que no caso das simulações da distribuição de Gibbs em redes com condições periódicas de contorno, deve ser o de uma reta ascendente, em concor dância com a teoria de escala de tamanho finito ( Landau 1976). A reta da figura 12 é a interpolação dos pontos por meio de regressão linear, e vemos que os pontos não se ajustam, aparentemente, em uma reta. Se acreditarmos que esta reta deva representar a forma da curva o valor de $T c$ será da ordem de 2.8. O valor de $T_{c}$ que achamos mais consistente com os dados é da ordem de 2.7 , aproximadamente de acordo com a forma do gráfico e com uma interpolação por um polinômio de ordem $2 \mathrm{em} \mathrm{N}^{-1}$. Entretanto não seguimos em um esforço maior para ajustar a curva $T_{c} \times \mathrm{N}^{-1}$ devido ao fato de hão possuirmos uma teoria para sua forma. Para termos de comparação, lembramos que o valor exato de $T_{c}$ é $2.269 \ldots$, enquanto que em campo médio $T_{c}=4.0$, na aproximação de Bethe $T_{c}=2.88 \ldots$..e na aproximação, já comentada, de resolvermos o sistema de equações

$$
m_{i}=\sum_{\left\{\sigma_{j}\right\}} \tanh \left[\beta J \sum_{j} \sigma_{j}\right] \prod_{j} \frac{1}{2}\left(1+\sigma_{j} m_{j}\right),
$$

impondo condições de invariância translacional, com todos os $m_{i}$ iguais, e adotando condições de contorno periódicas (Apêndice 2), o valor sugerido de $T_{c}$ é $3.089 \ldots \mathrm{Na}$ figura 12 admitimos que a incerteza em $T_{c}$ está entre 0.04 ( intervalo de temperaturas em nossas corrridas), para as redes maiores, e 0.08 para as menores. Essas incertezas são consistentes com os erros nos valores dos máximos dos calores específicos. 


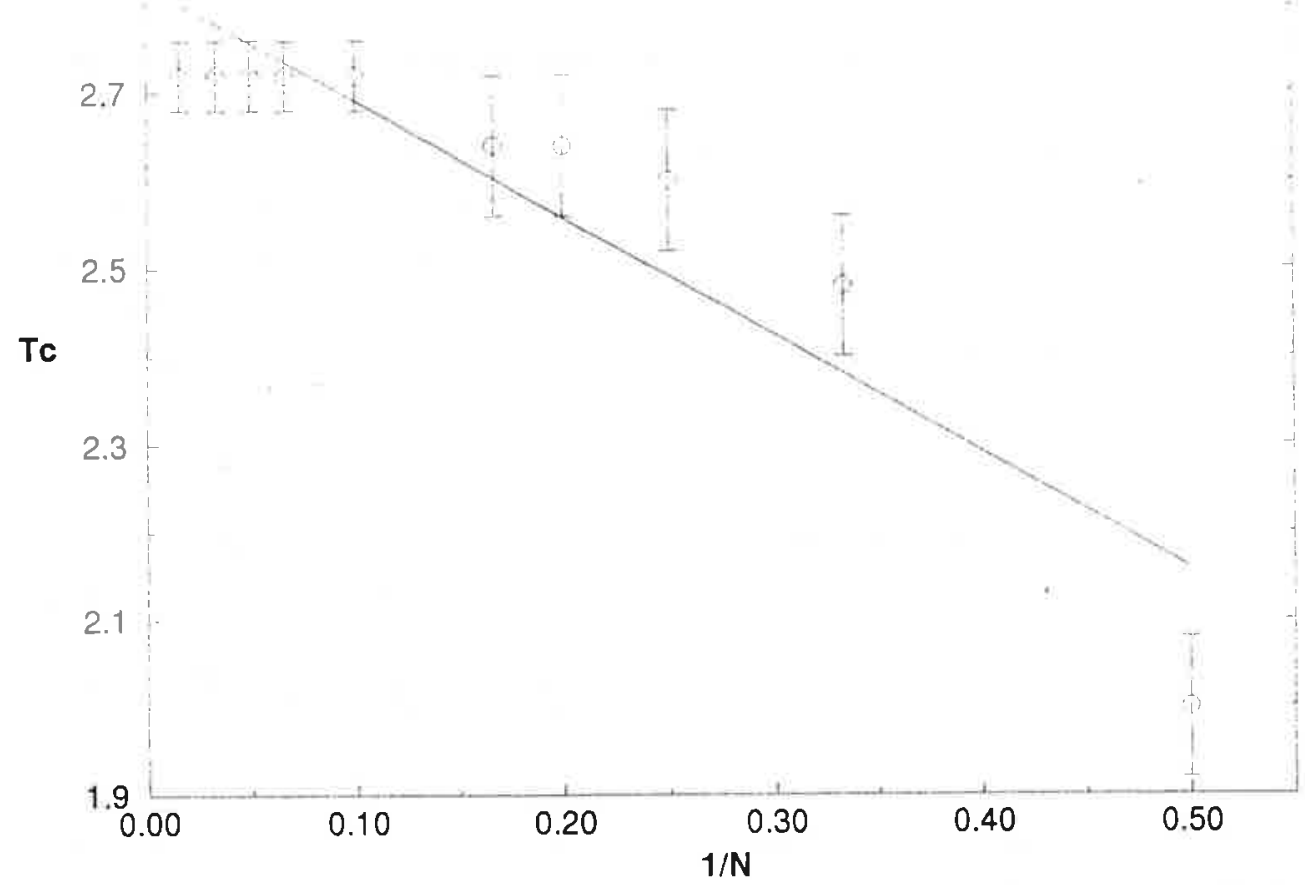

Fig.12

Observemos que a estimativa de $T_{c}(\mathrm{~N} \rightarrow \infty)$ ( entre 2.7 e 2.8 ) contraria, aparentemente, a perspectiva que os autores têm de seu método, uma vez que, como ponderamos no Apêndice 2, não há muitas razões para aceitar soluções não homogêneas ( $m_{i}$ diferente sítio a sítio) no caso da aplicação do sistema de equações acima à rede quadrada ferromagnética de tamanho qualquer. Lembramos, a esse respeito, que também fizemos simulações com uma rede 2 × 2 ( tratada no Apêndice 2 com mais detalhe pela aplicação do sistema de equações citado ) sem encontrar uma boa correspondência com a interpretação dos autores sobre o método. Mesmo as curvas da Fig.01, de magnetização em função de $T$, segundo a definição de magnetização dos autores, não sugerem essa interpretação . Como última observação a esse respeito, parece-nos difícil conciliar a visão de Netz e Berker sobre seu algoritmo com os efeitos de tamanho finito que observamos em sua aplicação, dado que aplicando o sistema de equações acima a redes de qualquer tamanho ( com condições de homogeneidade) teremos sempre como resposta o mesmo valor de $T_{c}$ ( $3.089 \ldots$... 


\section{IV - A Aplicação do Algortitmo de Netz e Berker ao Modelo de Blume-Capel}

\section{IV.1 - Apresentação do Modelo de Blume-Capel}

Originalmente, o modelo de Blume-Capel foi criado para explicar o comportamento de certos materiais magnéticos, como o $\mathrm{UO}_{2}$, capazes, a campo nulo, de sofrer transições de fase tanto de primeira ordem quanto de segunda (Blume, 1966, Capel, 1966). Há generalizações desse modelo inclusive em versões de gás na rede, das quais a mais famosa é a que explica a transição $\lambda$ e a separação de fases superluida e normal em misturas $\mathrm{He}_{e}{ }^{3}-\mathrm{H}_{e}{ }^{4}$, proposta por Blume, Emery e Griffiths (Blume, 1971). Vamos apresentar aqui sucintamente as caractetísticas essenciais do modelo de Blume-Capel em sua versão para magnetos.

Consideremos uma rede em cujos sítios colocamos sinais $s_{i}$ que podem ser $0,+1$ e -1 . A energia desse sistema de "spins 1 " será

$$
\mathrm{H}=-J \sum_{<i j>} s_{i} s_{j}+\Delta \sum_{i} s_{i}^{2}-h \sum_{i} s_{i} \quad(J e \Delta>0),
$$

onde o primeiro termo representa a interação "de troca", com a soma se estendendo a todos os pares de sítios primeiros vizinhos e com o terceiro representando o efeito de Zeeman, análogamente ao caso ferromagnético de Ising. O segundo termo, que vai introduzir a diferença, levando à existência possível de transições de primeira ordem, pode ser encarado como representando, em cada ion (sítio), uma separação entre estados $S_{Z}=0$ e $S_{Z}= \pm 1$ de um tripleto. A explicação desse termo, chamado de termo de campo cristalino, se encontra em textos de Física do Estado Sólido ( Kittel, 1967), ou nas referências originais de Blume e Capel.

É interessante encararmos a variável $\Delta$ como uma espécie de campo, conjugado à variável extensiva

$$
\sum_{i} s_{i}^{2}
$$

da mesma forma que o campo magnético $h$ é conjugado a

$$
\sum_{i} s_{i}
$$

que é o "momento magnético" adimensional total de uma configuração $\{s\}$. Dessa maneira podemos pensar, a campo nulo, no plano $D \times T, \operatorname{com} D=\Delta / J$ e $T=1 / \beta J$ (variáveis adimensionais convenientes) como um plano de fases, como normalmente pensamos o plano $h \times T$ para um magneto ou, ainda, o plano de fases pressão $\mathrm{x}$ temperatura para um fluido. Vamos pensar agora os extre$\operatorname{mos} D=0$ e $T=0$ desse plano.

Quando $D=0$ temos um ferromagneto. A campo $h$ nulo, a transição deverá ser então de segunda ordem. No outro extremo, $T=0$, analisamos o estado fundamental do modelo. Novamente a campo nulo, que é a situação que trataremos pela aplicação do algoritmo NB, poderemos ter duas possibilidades para o estado fundamental:

a) todos os spins estão alinhados, não importando a direção, uma vez que se trocamos os sinais de todos os spins a energia da nova configuração não se alterará. Nas unidades adimensionais definidas , a energia admensional por sítio será $D-q / 2, \operatorname{com} q$ sendo o número de coordenação da rede.

b) todos os $s_{i}$ são nulos. A energia desse estado é 0 .

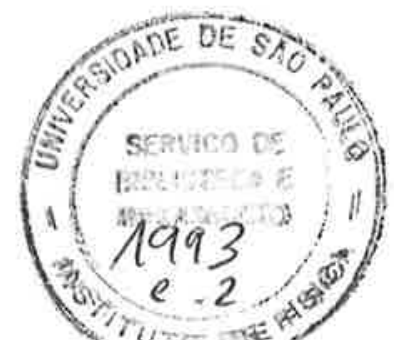


A situação a) é favorecida se $D<q / 2$. enquanto b) será favorecida para $D>q / 2$. Assim, teremos uma transição de fase de primeira ordem em $D=q / 2$.

Do que dissemos acima, já podemos esperar a existência de transições tanto de segunda ordem como de primeira no modelo de Blume-Capel. De fato, antecipando um pouco os resultados desse modelo, sabemos que no plano $D \times T$ há duas linhas de transição, uma de primeira ordem e uma de segunda, que se encontram tangencialmente em um ponto chamado tricrítico. A linha de segunda ordem deve terminar em algum ponto do eixo $T, \operatorname{com} D=0$, e a de primeira no ponto $D=q / 2$ e $T=0$. O ponto de encontro das duas linhas tem seu nome devido ao fato de ser um ponto onde, considerando o espaço de fases completo $D, T, h^{\prime}=h / J$, terminam três linhas críticas de transição (Blume, 1971).

A forma geral do diagrama de fases a campo nulo pode ser obtida em teoria de campo médio, em seu sentido variacional, por meio da hamiltoniana de teste

$$
\mathrm{H}_{\mathrm{o}}=-\eta J \sum_{i} s_{i}+D J \sum_{i} s_{i}^{2}
$$

Dessa forma, o limite superior da função de Gibbs fica

$$
\Phi(\eta)=-\frac{1}{\beta} \ln Z_{o}+\left\langle H-H_{0}\right\rangle_{0} .
$$

Esse limite superior pode ser minimizado em relação ao parâmetro livre $\eta$, o que fixará uma equação de consistência para o valor de $\left\langle s_{i}\right\rangle \equiv m$. Da mesma maneira que no caso já estudado do Modelo de Ising, teremos $\eta=\sum_{j}<s_{j}>=q m$, de maneira que é possivel obter uma expansão de $\Phi$ em potências de $m$, isto é, obter uma teoria de Landau para o modelo*. Observamos que o campo $\eta$ homogêneo em $\mathrm{H}_{\circ}$ pode também variar de sítio a sítio, como havíamos feito no caso Ising. Isto será usado na seção seguinte para aplicar o algoritmo de Netz e Berker ao modelo agora em questão. me, 1971).

\footnotetext{
* Os detalhes essenciais desse cálculo se encontram no artigo de Blume, Emery e Griffiths ( Blu-
} 


\section{IV.2 - A obtenção da prescrição de Netz e Berker para o modelo de Blume-Ca-}

pel.

Como já havíamos dito na seção anterior, podemos usar campos $\eta_{i}$ inomogêneos na hamiltoniana de teste $\mathrm{H}_{\mathrm{o}}$ usada no cálculo de campo médio variacional para o modelo de Blume-Capel. Examinemos um pouco a situação, considerando agora nulo o campo externo. Temos

$$
H_{0}=-\sum_{i} J \eta_{i} s_{i}+\Delta \sum_{i} s_{i}^{2}
$$

de forma que

$$
\mathrm{Z}_{0}=\prod_{i}\left[1+2 e^{-D / T} \cosh \left(\eta_{i} / T\right)\right]
$$

onde $D=\Delta / J$ e $T=1 / \beta J$. O funcional a ser mimimizado é dado por

$$
\Phi\left(\left\{\eta_{i}\right\}\right)=-J T \sum_{i} \ln \left[1+2 e^{-D / T} \cosh \left(\eta_{i} / T\right)\right]-J \sum_{<i j>}<s_{i} s_{j}>_{0}+J \sum_{i} \eta_{i}<s_{i}>_{0},
$$

onde

$$
\begin{aligned}
<s_{i}>_{0} & =\frac{\sum_{s\}} s_{i} e^{\beta J} \sum_{i} \eta_{i} s_{i}-\beta \Delta \sum_{i} s_{i}}{\sum_{\{s\}} e^{\beta J} \sum_{i} \eta_{i} s_{i}-\beta \Delta \sum_{i} s_{i}} \\
= & \frac{2 e^{-D / T} \sinh \left(\eta_{i} / T\right)}{1+2 e^{-D / T} \cosh \left(\eta_{i} / T\right)},
\end{aligned}
$$

e

$$
\begin{aligned}
& <s_{i} s_{j}>_{0}=\frac{\sum_{\{s\}} s_{i} s_{j} e^{\beta J \sum_{i} \eta_{i} s_{i}-\beta \Delta \sum_{i} s_{i}}}{\sum_{\{s\}} e^{\beta J} \sum_{i} \eta_{i} s_{i}-\beta \Delta \sum_{i} s_{i}} \\
& =\left\langle s_{i}\right\rangle_{0}\left\langle s_{j}\right\rangle_{0}=\frac{2 e^{-D / T} \sinh \left(\eta_{i} / T\right)}{1+2 e^{-D / T} \cosh \left(\eta_{i} / T\right)} \frac{2 e^{-D / T} \sinh \left(\eta_{j} / T\right)}{1+2 e^{-D / T} \cosh \left(\eta_{j} / T\right)} .
\end{aligned}
$$

Aplicando agora a condição de extremização de $\Phi\left(\left\{\eta_{i}\right\}\right)$ temos

$$
\eta_{i}=\sum_{j} \frac{2 e^{-D / T} \sinh \left(\eta_{j} / T\right)}{1+2 e^{-D / T} \cosh \left(\eta_{j} / T\right)}
$$

ou seja

$$
\eta_{i}=\sum_{j}\left\langle s_{j}\right\rangle_{0}
$$


Voltando à definição de $\left\langle s_{i}\right\rangle_{0}$, temos $\mathrm{N}^{\mathrm{d}}$ equaçōes de consistência;

$$
\left\langle s_{i}>_{0}=\frac{2 e^{-D / T} \sinh \left[\left(\sum_{j}<s_{j}>_{0}\right) / T\right]}{1+2 e^{-D / T} \cosh \left[\left(\sum_{j}<s_{j}>_{0}\right) / T\right]} .\right.
$$

De maneira semelhante ao ocorrido no modelo de Ising, $\left\langle s_{i}\right\rangle_{0}$ representa $\left\langle s_{i}\right\rangle$ na aproximação de campo médio.

Dadas as considerações acima, estamos em condições agora de formular o algoritmo de NB para o modelo de Blume-Capel. A idéia é, então, atualizar um valor de magnetização local $m_{i}$ por meio da função

$$
m_{i}=\frac{2 e^{-D / T} \sinh \left(\eta_{i} / T\right)}{1+2 e^{-D / T} \cosh \left(\eta_{i} / T\right)},
$$

onde o campo de troca local $\eta_{i}=\sum_{j} s_{j}$ será construído com os $s_{j}$ dados com probabilidade

$$
\omega_{j}\left(s_{j}\right)=\frac{e^{s_{j} \eta_{j} / T-D s_{j}^{2} / T}}{1+2 e^{-D / T} \cosh \left(\eta_{j} / T\right)} .
$$

A forma de $\omega_{j}\left(s_{j}\right)$ foi escolhida de maneira a ser formalmente idêntica à distribuição de probabilidades de $s_{j}$ dada pela hamiltoniana de teste $\mathrm{H}_{0}$. A diferença, como já havíamos notado no caso do modelo de Ising, é que $\eta_{j}$ será agora o valor da soma dos spins, vizinhos ao sítio $j$, dada na última ocasião em que este sítio foi visitado. 


\section{IV.3 - A Implementação do algoritmo}

Na aplicação do algoritmo, da mesma forma que no ferromagneto, fizemos o uso de condições periódicas de contorno. Também aqui usamos uma versão sequencial onde um passo de Monte Carlo é uma varrida completa sobre a rede. Podemos iniciar o cálculo de uma grandeza, dados $D$ e $T$, colocando nos sítios $i$ valores iniciais de $\eta_{i}$ ( no caso de uma rede quadrada, há 9 possíveis valores de $\eta_{i}=\sum_{j} s_{j}$ ). A iteração em um sítio $i$ consistirá dos seguintes passos:

a) localizado o sítio $i$, procuramos seus sítios vizinhos. Escolhemos algum destes, $j$. b)colocamos em $s_{j}$ o sinal +1 e sorteamos um número aleatório entre 0 e 1 .

c) calculamos a probabilidade de $s_{j}$ ser 0 , dada por

$\omega_{j}(0)=\frac{1}{1+2 e^{-D / T} \cosh \left(\eta_{j} / T\right)}$.

Se o número aleatório é menor do que $\omega_{j}(0)$ então colocamos, $\mathrm{em} j, s_{j}=0$.

d) calculamos a soma das probabilidades do spin $s_{j}$ passar a 0 ou a 1 , dada por

$\omega_{j}\left(s_{j}=0\right.$ ou $\left.s_{j}=1\right)=\omega_{j}(0)+\omega_{j}(+1)$,

com $\omega_{j}(0)$ já conhecido e com

$\omega_{j}(+1)=\frac{e^{\eta_{j} / T-D / T}}{1+2 e^{-D / T} \cosh \left(\eta_{j} / T\right)}$.

Se o número aleatório que sorteamos for maior do que $\omega_{j}\left(s_{j}=0 o u s_{j}=1\right)$, então colocaremos em $s_{j}$ o valor -1 .

e) Repetimos os passos a), b) e c) para todos os vizinhos de $i$ e atualizamos $\eta_{i}$ pela soma desses spins e $m_{i}$ por $m_{i}=\frac{2 e^{-D / T} \sinh \left(\eta_{i} / T\right)}{1+2 e^{-D / T} \cosh \left(\eta_{i} / T\right)}$.

Como já dissemos, varremos, em um PMC, a rede toda fazendo essas atualizações sítio a sítio, Em cada passo, guardamos o valor de uma grandeza de interesse e , dado um certo número de PMC, calculamos uma média. Como mostraremos na próxima seção, a grandeza que mais utiliza$\operatorname{mos}$ foi $\frac{\left(\sum_{i} s_{i}^{2}\right)}{(\mathrm{N} \times \mathrm{N})}$, para a rede quadrada de $\mathrm{N}^{2}$ spins, pois essa é a grandeza que usamos para nos decidir sobre a ordem de uma transição. A forma geral do algoritmo, desenvolvida por nós para redes quadradas, é muito semelhante à apresentada no caso do ferromagneto, com as diferenças ficando apenas restritas à prescrição para a atualização do campo local em um sítio dado. 
IV.4 - A forma geral das curvas de magnetização e $Q \equiv\left\langle\left(\sum_{i} s_{i}^{2}\right) /(N \times N)\right\rangle$ em redes quadradas

Primeiramente, devemos lembrar que todos os resultados aqui apresentados para o modelo de Blume-Capel são qualitativos, pois não foi feita nenhuma análise de erros. Usamos uma rede de 30 x 30 sítios, com 500 PMCs descontando os 100 primeiros. Para termos uma idéia comparativa, a amostragem usada por B. L. Arora e D.P. Landau, em um trabalho sobre o modelo em questão, usando um algoritmo de Metrópolis, foi de 100 até 2000 PMCs para redes de tamanho variando entre $8 \times 8$ e $60 \times 60$, com o descarte dos primeiros 20 até 50 PMCs ( Arora, 1971). As Figs. 13 e 14 mostram a variação, respectivamente, do módulo da magnetização, $M=\left|\left\langle\left(\sum_{i} s_{i}^{2}\right) /(\mathrm{N} \times \mathrm{N})\right\rangle\right|$ e de $Q=\left\langle\left(\sum_{i} s_{i}^{2}\right) /(\mathrm{NxN})\right\rangle$, para a temperatura $T \equiv 1 / \beta J=0.5$. Um círculo nessas figuras indica uma corrida iniciando do valor $D=0$ com todos os $\eta_{i}=4$, enquanto que com quadrados assinalamos uma corrida partindo de $\quad D=3$ com todos os $\eta_{i}=0$ e reduzindo sucessivamente $D . O$ fato de as curvas não coincidirem e, além disso, a existência de saltos grandes, é indicativo de que a transição deve ser de primeira ordem.

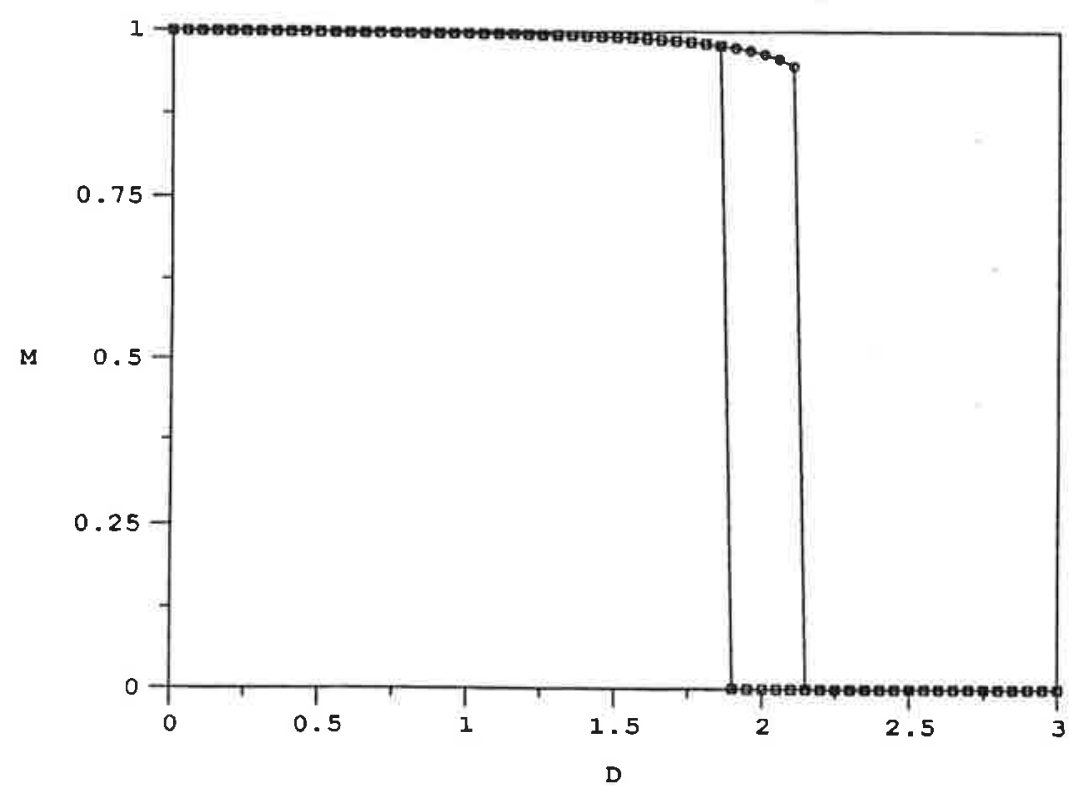

Fig.13 


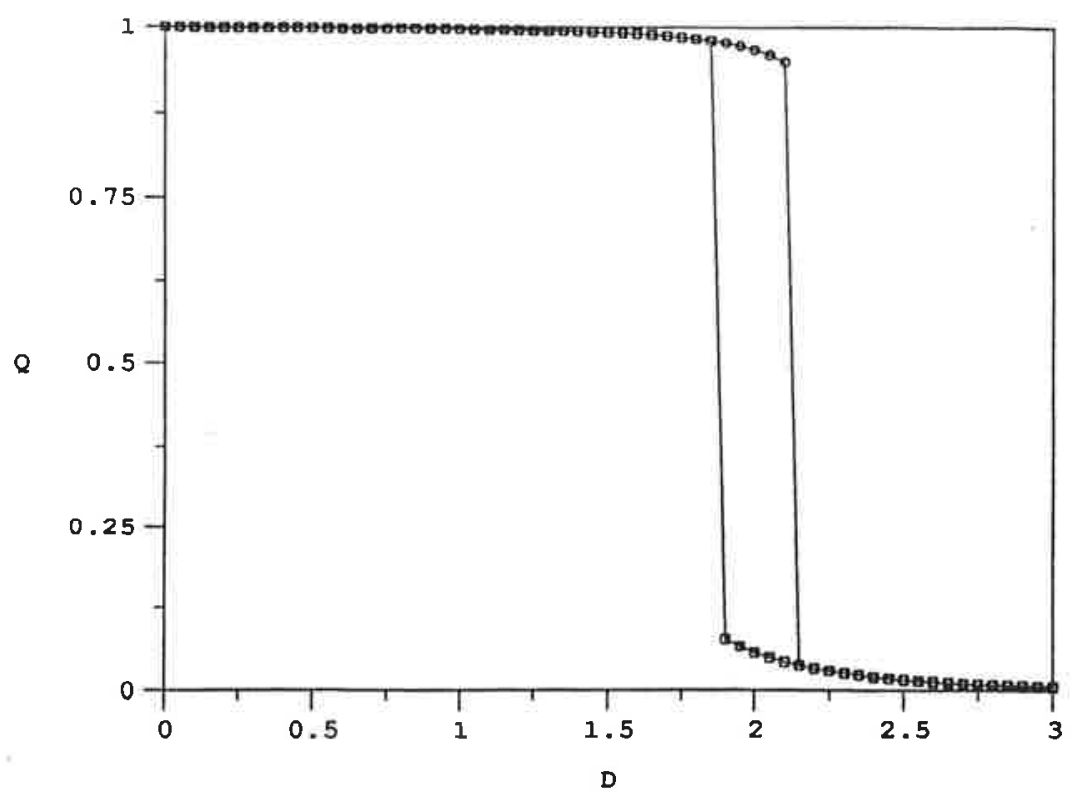

Fig.14

Observemos, por outro lado, nas Figs. 15 e 16 abaixo e seguinte, a variação de $M$ e $Q$ com $T$ para $D=0$.

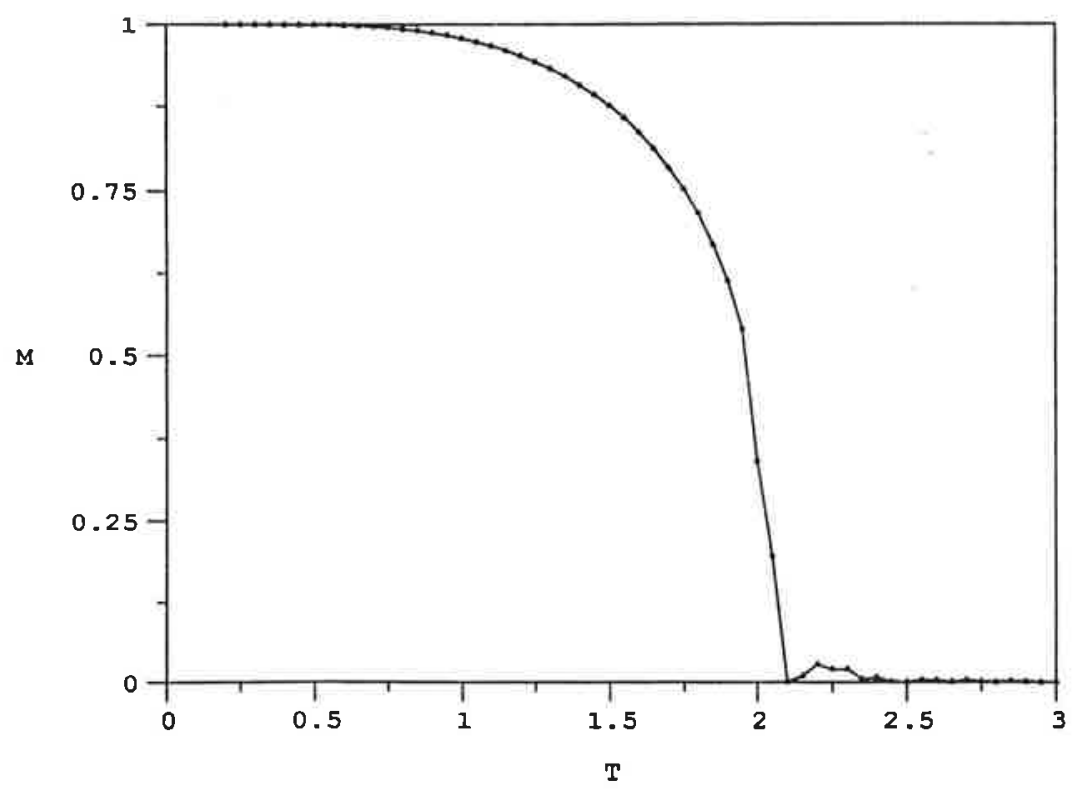

Fig.15 


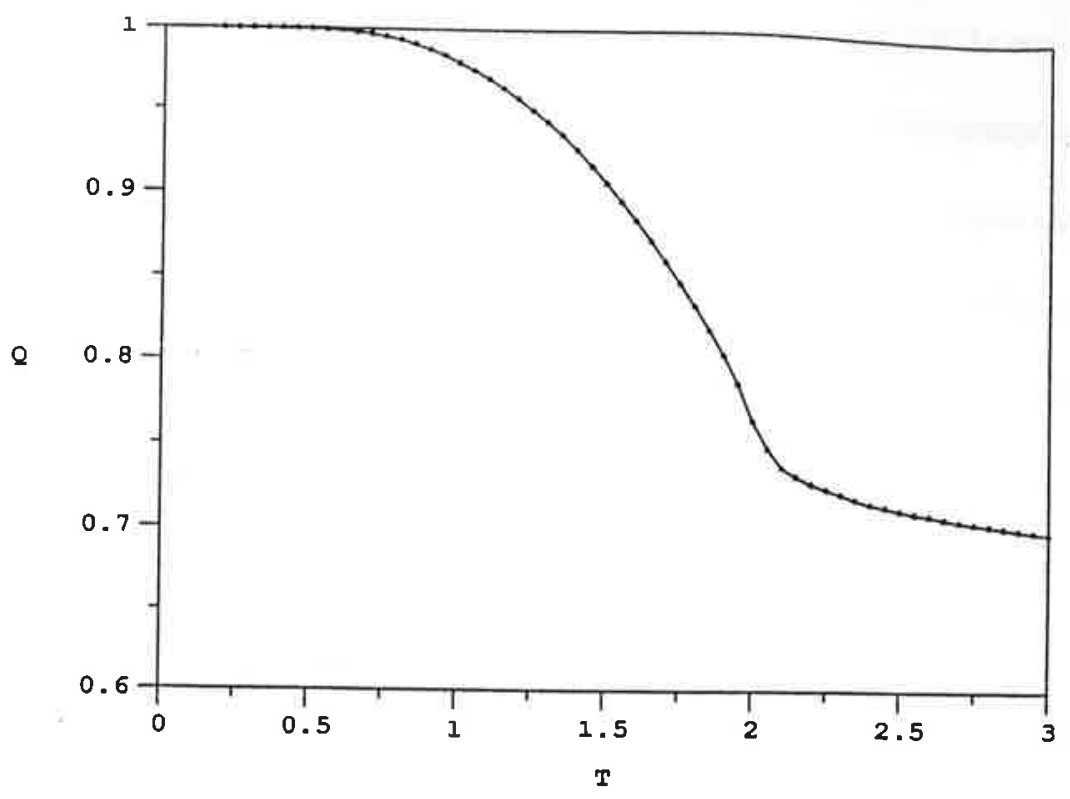

Fig.16

As curvas acima podem ser consideradas típicas de uma transição de segunda ordem, uma vez que não sentimos a existência de nenhum salto nos valores das grandezas, não havendo razões para se suspeitar de histerese, como ocorrido no caso $T=0.5$, visto anteriormente.

Aparentemente as figuras nesta seção estão indicando que um comportamento anômalo de $M$ sempre é acompanhado de um comportamento anômalo em $Q$. Na verdade aplicaremos, na seção seguinte, o princípio de que uma anomalia nas funções termodinẩmicas se reflete em suas derivadas, que são as equações de estado, para indicar os pontos de transição no plano $D \times T$. Para isso usaremos sempre a grandeza $Q$, sujeita a flutuações menores do que $M \mathrm{e}$, portanto, mais adequada às análises numéricas. 
de fases.

O diagrama de fases foi esboçado varrendo-se o plano $D \times T$ da seguinte forma:

a) varremos isotermas a diferentes temperaturas começando sempre de $D=0$ com os $\eta_{i}=4$.

Na Fig. 17 os pontos marcados com quadrados representam essas simulações.

b) varremos isotermas a diferentes temperaturas começando sempre de $D=3$ e com $\eta_{\mathrm{i}}=0$.

Os pontos, na Fig. 17, marcados com losangos representam essas simulações.

Todos os pontos da Fig.17, para $T>0.75$, que devem representar transições de segunda ordem, foram tirados do máximo da variação de $Q$ em relação a $D$. Para $T<0.75$, região onde deve haver transições de primeira ordem, os pontos assinalados por quadrado e losangos são pontos onde $Q$ dá um grande salto " para baixo" (quadrados) ou " para cima" (losangos). Nessa região, um único ponto de transição de primeira ordem foi calculado, estando marcado na figura por um círculo. Mostraremos em seguida como este ponto foi obtido, mas, antes, observemos que apesar de não conseguirmos uma boa coincidência entre os pontos marcados com quadrados e losangos para $T$, aconteceu uma ocasião onde houve inversão da ordem dos caracteres (na temperatura $T=1.75$ ) indicando que nessa região a transição deve mesmo ser de segunda ordem. Na verdade, a região de segunda ordem deve ser mais facilmente trabalhada com simulações a $D$ constante, mas nosso objetivo é aqui mais qualitativo - queremos saber ser o algoritmo é capaz de distinguir a ordem de uma transição.

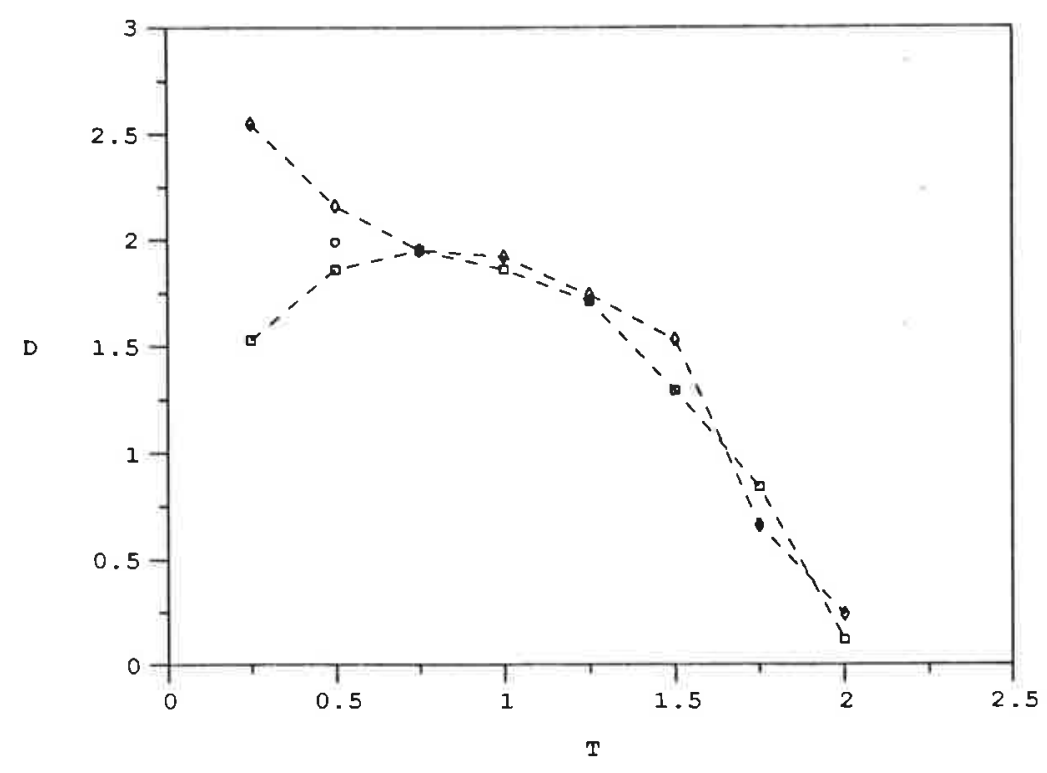

Flg.17

Para entendermos como foi obtido o valor de $D$ de transição de primeira ordem em $T=0.5$ lembramos, primeiramente, que

$$
Q=\frac{\partial G}{\partial D},
$$

onde $G$ é a energia livre por sítio, em unidades de $J$ e a campo nulo. No ponto de transição, $G$ deve ter um cotovelo, indicando a indefinição de $Q$ entre dois valores bem distintos. Se possuirmos algu- 


\section{IV.5 - A localização dos pontos de transição e o levantamento do dlagrama} de fases.

O diagrama de fases foi esboçado varrendo-se o plano $D \times T$ da seguinte forma:

a) varremos isotermas a diferentes temperaturas começando sempre de $D=0$ com os $\eta_{i}=4$.

Na Fig. 17 os pontos marcados com quadrados representam essas simulaçōes.

b) varremos isotermas a diferentes temperaturas começando sempre de $D=3$ e com $\eta_{\mathrm{i}}=0$.

Os pontos, na Fig. 17, marcados com losangos representam essas simulações.

Todos os pontos da Fig.17, para $T>0.75$, que devem representar transições de segunda ordem, foram tirados do máximo da variação de $Q$ em relação a $D$. Para $T<0.75$, região onde deve haver transições de primeira ordem, os pontos assinalados por quadrado e losangos são pontos onde $Q$ dá um grande salto " para baixo" (quadrados) ou " para cima" (losangos). Nessa região, um único ponto de transição de primeira ordem foi calculado, estando marcado na figura por um círculo. Mostraremos em seguida como este ponto foi obtido, mas, antes, observemos que apesar de não conseguirmos uma boa coincidência entre os pontos marcados com quadrados e losangos para $T$, aconteceu uma ocasião onde houve inversão da ordem dos caracteres (na temperatura $T=1.75$ ) indicando que nessa região a transição deve mesmo ser de segunda ordem. Na verdade, a região de segunda ordem deve ser mais facilmente trabalhada com simulações a $D$ constante, mas nosso objetivo é aqui mais qualitativo - queremos saber ser o algoritmo é capaz de distinguir a ordem de uma transição.

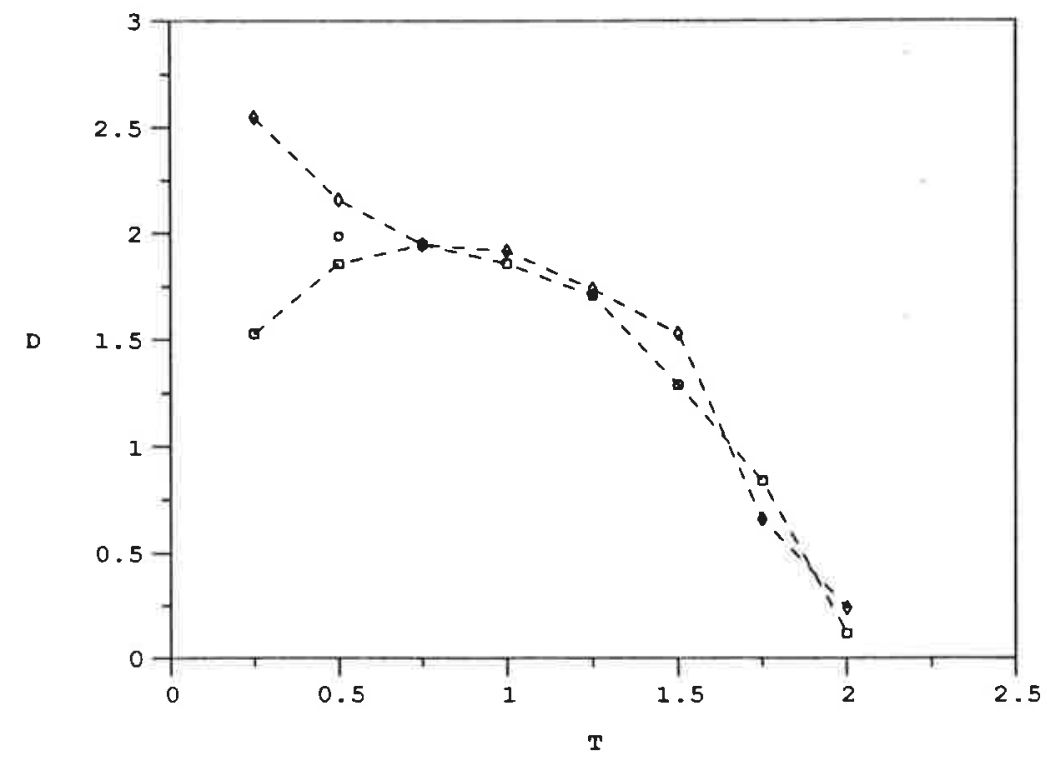

Fig.17

Para entendermos como foi obtido o valor de $D$ de transição de primeira ordem em $T=0.5$ lembramos, primeiramente, que

$$
Q=\frac{\partial G}{\partial D}
$$

onde $G$ é a energia livre por sítio, em unidades de $J$ e a campo nulo. No ponto de transição, $G$ deve ter um cotovelo, indicando a indefinição de $Q$ entre dois valores bem distintos. Se possuirmos algu- 
mas referências para o cálculo de $G$, poderemos obter o valor $D$ to na transição de primeira ordem à temperatura $T_{o}$. No ponto de coordenadas $\left(T_{o}, D=0\right)$ do plano $D \times T$, a energia livre é dada por

$$
G\left(T_{o}, D=0\right)=E\left(T_{o}\right)-T_{o} S\left(T_{o}\right),
$$

onde $E$ e $S$ representam a energia por sítio em unidades de $J$ e a entropia, também por sítio, em unidades de $K_{B} . E$ ( $\left.T_{0}\right)$ é calculável diretamente pela aplicação do algoritmo, enquanto $S\left(T_{0}\right)$ é calculável por

$$
S\left(T_{o}\right)=\frac{\ln 2}{\mathrm{~N}^{2}}+\left(\int_{0}^{T_{o}} \frac{C}{T} d T\right)_{D=0}
$$

onde $\mathrm{N}^{2}$ é o número de sítios, $\ln 2 / \mathrm{N}^{2}$ é a entropia em $T=0 \operatorname{com} D=0$ e $C$ o calor específico adimensional, $d E / d T$, que pode ser obtido de dados de energia $\mathrm{x}$ temperatura para $D=0$. Indo agora ao outro extremo, o ponto $\left(T_{o}, D \rightarrow \infty\right), G$ deve se anular, pois devemos $\operatorname{ter} E\left(T_{o}, D \rightarrow \infty\right)=0$, ( fase paramagnética ) e também $S=\left(T_{o}, D \rightarrow \infty\right)=0$, pois a entropia em $T=0$ e $D=\rightarrow \infty$ é 0 (só há um estado) e ainda

$$
\left(\int_{0}^{T_{0}} \frac{C}{T} d T\right)_{D \rightarrow \infty} \rightarrow 0
$$

pois $C(T, D \rightarrow \infty) \rightarrow 0$ ( $E$ é constante e nula) dentro do limite da integral. Assim, temos nossas referências. Dada a relação entre $Q$ e $G$ teremos

$$
\begin{aligned}
\int_{D \rightarrow \infty}^{D_{T_{o}}} Q\left(T_{o}, D\right) d D & =G\left(T_{o}, D T_{o}\right)-G\left(T_{o}, D \rightarrow \infty\right) \\
& =G\left(T_{o}, D T_{o}\right)
\end{aligned}
$$

e

$$
\begin{aligned}
\int_{o}^{D_{T_{o}}} Q\left(T_{o}, D\right) d D & =G\left(T_{o}, D T_{o}\right)-G\left(T_{o}, D=0\right) \\
& =G\left(T_{o}, D_{T_{o}}\right)-E\left(T_{o}\right)+T_{o} \frac{\ln 2}{\mathrm{~N}^{2}}+T_{o}\left(\int_{0}^{T_{o}} \frac{C}{T} d T\right)_{D=0}
\end{aligned}
$$

de onde obtemos uma equação para $D_{T_{o}}$ na forma

$$
\int_{0}^{D_{T_{o}}} Q\left(T_{o}, D\right) d D=\int_{D \rightarrow \infty}^{D_{T_{o}}} Q\left(T_{o}, D\right) d D-E\left(T_{o}\right)+T_{o} \frac{\ln 2}{\mathrm{~N}^{2}}+T_{o}\left(\int_{0}^{T_{o}} \frac{C}{T} d T\right)_{D=0}
$$

Na Fig.18, a linha de pontos marcados com losangos representa o lado esquerdo da equação acima, e a linha com os pontos em triângulos o lado direito. O ponto de intersecção representa a solução da equação, e está marcado, como dissemos, com um círculo na Fig.17. Para $D=3$ já podemos nos considerar no limite $D \rightarrow \infty$. Devemos lembrar que os métodos usados aqui para o cálculo de $G$ são conhecidos na literatura ( Binder, 1981). 


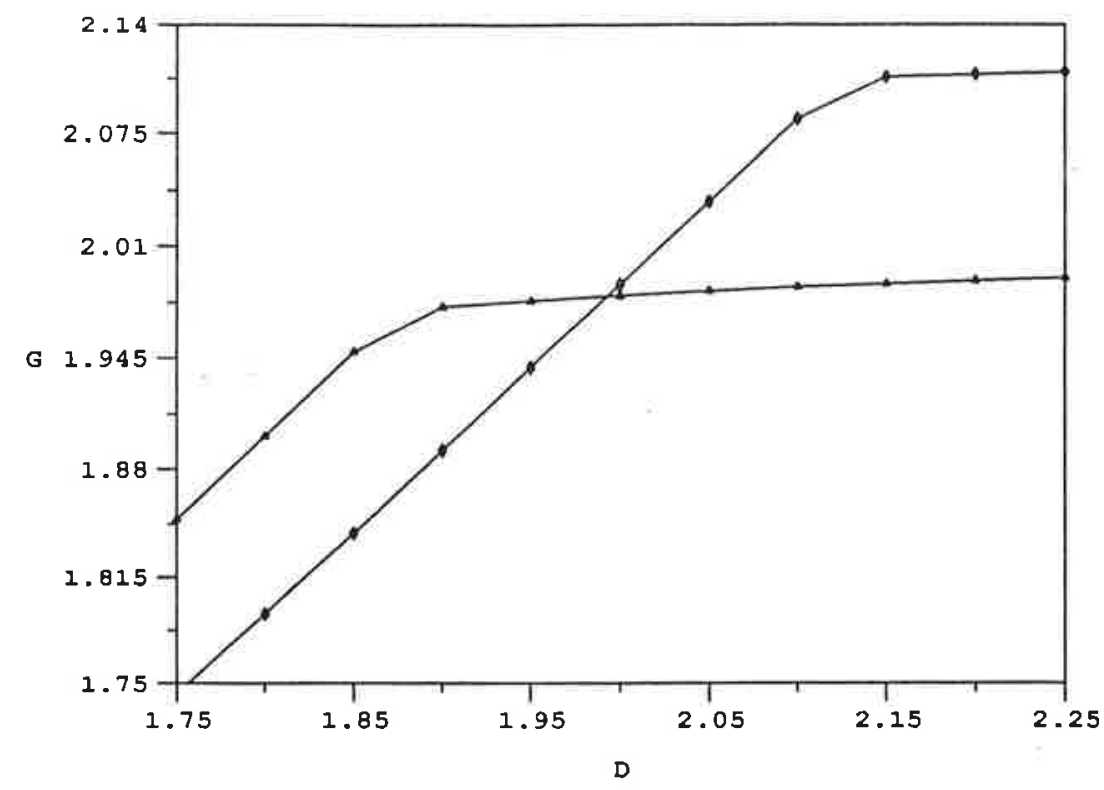

Fig.18

A Fig.19 indica uma comparação entre dados aproximados obtidos pelo algoritmo NB, para a linha de segunda ordem, com os resultados de outras abordagens. A linha cheia representa a aproximação de campo médio. A linha pontilhada representa a aproximação de Bethe-Peierls para uma rede quadrada (Oliveira, 1985). Os pontos indicados por triângulos correspondem ao próprio algoritmo NB, e os assinalados por círculos, os dados de Arora e Landau, obtidos de seu trabalho já comentado ( simulações da distribuição de Gibbs).

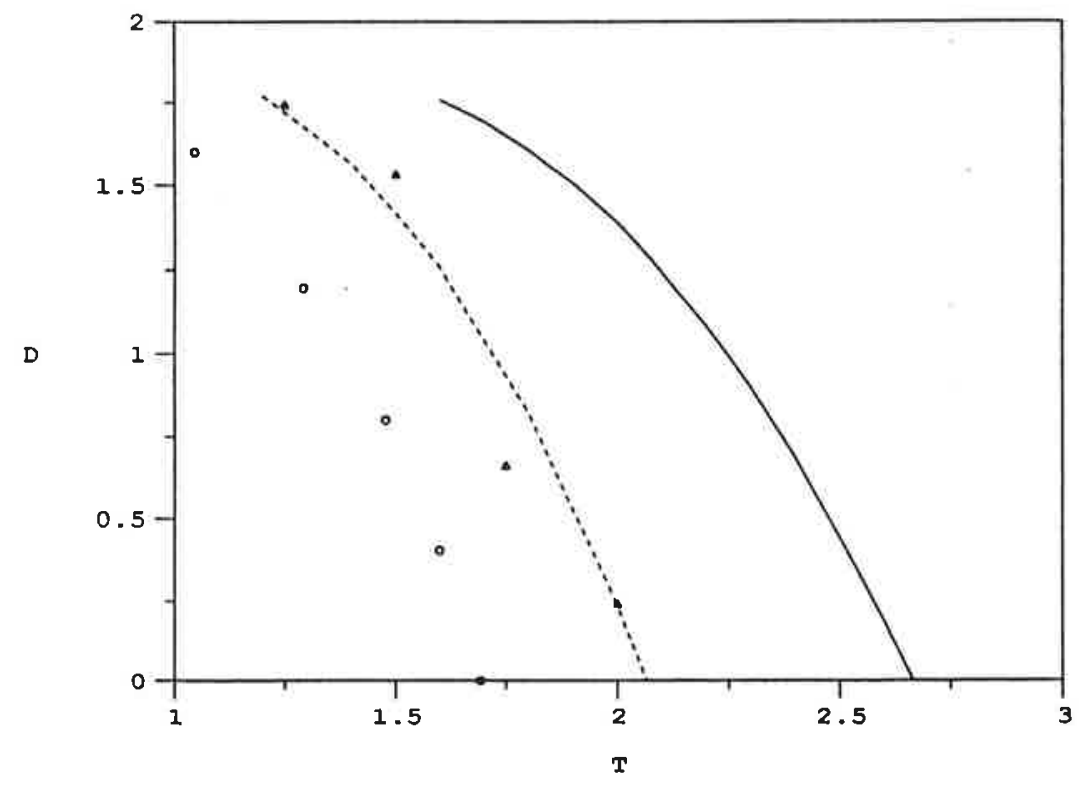

FIg.19

Apesar de não estarmos muito certos sobre a eficiência do algoritmo de NB aplicado ao modelo de Blume-Capel, podemos observar que seus resultados são muito semelhantes à abordagem de Bethe-Peierls. 


\section{V - Conclusão}

Em sua aplicação aos modelos estudados neste trabalho o algoritmo de Netz e Berker se revelou capaz de produzir resultados qualitativamente corretos, comparáveis a uma aproximação de Bethe-Peierls. No entanto, com um esforço computacional equivalente, é possível utilizar os métodos convencionais de Monte Carlo para obter resultados muito mais realistas. Como as aproximações de Bethe-Peierls em geral fornecem expressões analíticas, torna-se difícil justificar a utilização do algoritmo de Netz e Berker em quaisquer problemas de interesse físico.

É difícil perceber o que o algoritmo de Netz e Berker está realmente fazendo. Certamente ele não é equivalente a uma solução das equações gerais de campo médio. Também não parece ser equivalente a uma solução das equações de consistência advindas de uma fatorização das probabilidades dos estados de spin, como foi sugerido por Banavar, Cieplak e Maritan ( Banavar, 1991) e aceito por Netz e Berker (Netz, 1991 b). Embora não tenhamos estudado em detalhe a dinâmica produzida pelo algoritmo, há claras indicações de que ele não obedece ao princípio do balanço detalhado e nem conduz, no equilíbrio, a um estado de Gibbs. 


\section{Apêndice 1}

Prova de que, na distribuição de Gibbs, $\left\langle\sigma_{i}\right\rangle=\left\langle\tanh \left[\beta J\left(\sum_{i} \sigma_{j}+h^{\prime}\right)\right]\right\rangle$, para o modelo de Ising.

\section{Temos}

$$
\begin{aligned}
& <\sigma_{i}>=\frac{\sum_{\{\sigma \mid} \sigma_{i} e^{\beta J\left(\sum_{<k j>} \sigma_{k} \sigma_{j}+h^{\prime} \sum_{k} \sigma_{k}\right)}}{\left.\sum_{\{\sigma\}} e^{\beta J\left(\sum_{<k j>} \sigma_{k} \sigma_{j}+h^{\prime}\right.} \sum_{k} \sigma_{k}\right)} \\
& \left.=\frac{1}{Z} \sum_{\{\boldsymbol{\sigma}\}} \sigma_{i} e^{\beta J \sigma_{i}\left(\sum_{j} \sigma_{j}+h^{\prime}\right)} e^{\beta J\left(\sum_{<\neq i j>} \sigma_{k} \sigma_{j}+h^{\prime}\right.} \sum_{k \neq i} \sigma_{k}\right) \\
& =\frac{1}{Z} \sum_{\left\{\sigma-\sigma_{i}\right\}}\left(\sum_{\sigma_{i}} \sigma_{i} e^{\beta J \sigma_{i}\left(\sum_{j} \sigma_{j}+h^{\prime}\right)}\right) e^{\beta J\left(\sum_{<\neq i j>} \sigma_{k} \sigma_{j}+h^{\prime} \sum_{k \neq i} \sigma_{k}\right)} \\
& =\frac{1}{Z} \sum_{\left\{\sigma-\sigma_{i}\right\}}\left(\frac{\sum_{\sigma_{i}} \sigma_{i} e^{\beta J \sigma_{i}\left(\sum_{j} \sigma_{j}+h^{\prime}\right)}}{\left.\sum_{\sigma_{i}} e^{\beta J \sigma_{i}\left(\sum_{j} \sigma_{j}+h^{\prime}\right)}\right)}\right)\left(\sum_{\sigma_{i}} e^{\beta J \sigma_{i}\left(\sum_{j} \sigma_{j}+h^{\prime}\right)}\right) e^{\beta J\left(\sum_{<\neq i j>} \sigma_{k} \sigma_{j}+h^{\prime} \sum_{k \neq i} \sigma_{K}\right)} \\
& =\frac{1}{Z} \sum_{\{\sigma\}} \tanh \left[\beta J\left(\sum_{j} \sigma_{j}+h^{\prime}\right)\right] e^{\beta J\left(\sum_{\langle k j\rangle} \sigma_{k} \sigma_{j}+h^{\prime} \sum_{k} \sigma_{k}\right)} \\
& =\left\langle\tanh \left[\beta J\left(\sum_{j} \sigma_{j}+h^{\prime}\right)\right]\right\rangle .
\end{aligned}
$$

A demonstração dessa relação se encontra também em Parisi ( Parisi, 1988 ). Ela é muitas vezes conhecida com identidade de Callen, e a aproximação de campo médio usual é obtida quando fazemos

$$
<\sigma_{i}>=\tanh \left[\beta J\left(\sum_{j}<\sigma_{j}>+h^{\prime}\right)\right] .
$$




\section{Apêndice 2}

Considerações sobre o sistema de equações sugerido como sendo equivalente ao algoritmo NB.

1) Equivalência entre as equações

$$
m_{i}=\sum_{\{\sigma j\}} \prod_{j} \frac{1}{2}\left(1+\sigma_{j} m_{j}\right) \tanh \left[\beta J\left(\sum_{j} \sigma_{j}+h^{\prime}\right)\right]
$$

a campo $h$ ' nulo, e o cálculo de $<\tanh \left[\beta J\left(\sum_{j} \sigma_{j}\right)\right]>$ por expansão de

$\tanh \left[\beta J\left(\sum_{j} \sigma_{j}\right)\right]$ nos produtos dos $\sigma_{j}$ com fatorização das médias.

Primeiramente, vamos tornar explícita a origem das equações de consistência acima. Do Apêndice 1 sabemos que, definindo $m_{i}$ como $\left\langle\sigma_{i}\right\rangle$,

$$
m_{i}=<\tanh \left[\beta J\left(\sum_{j} \sigma_{j}\right)\right]>\left(h^{\prime}=0\right)
$$

na distribuição de Gibbs. Calculemos porém a média na distribuição fatorizada

$$
P\{\sigma\}=\prod_{i} \frac{1}{2}\left(1+\sigma_{i} m_{i}\right)
$$

Teremos então

$$
\begin{aligned}
m_{i} \equiv\left\langle\sigma_{i}\right\rangle= & \sum_{\{\sigma\}} P\left\{\sigma \mid \tanh \left[\beta J\left(\sum_{j} \sigma_{j}\right)\right]\right. \\
= & \sum_{\sigma_{i}} \sum_{\sigma_{2}} \ldots \sum_{\left\{\sigma_{j}\right\}} \ldots \sum_{\sigma_{N}} \frac{1}{2}\left(1+\sigma_{1} m_{1}\right)\left(1+\sigma_{2} m_{2}\right) \ldots \\
& \ldots \prod_{j} \frac{1}{2}\left(1+\sigma_{j} m_{j}\right) \tanh \left[\beta J\left(\sum_{j} \sigma j\right)\right] \ldots \frac{1}{2}\left(1+\sigma_{N} m_{N}\right) \\
= & \sum_{\left\{\sigma_{j}\right\}} \prod_{j} \frac{1}{2}\left(1+\sigma_{j} m_{j}\right) \tanh \left[\beta J\left(\sum_{j} \sigma_{j}\right)\right] .
\end{aligned}
$$

Vamos agora desenvolver essa expressão para uma cadeia ferromagnética. Neste caso particular temos

$$
\begin{aligned}
<\sigma_{i}> & =\sum_{\sigma_{i-1}} \sum_{\sigma_{i+1}} \frac{1}{2}\left(1+\sigma_{i-1}<\sigma_{i-1}>\right) \frac{1}{2}\left(1+\sigma_{i+1}<\sigma_{i+1}>\right) \tanh \left[\beta J\left(\sigma_{i-1}+\sigma_{i+1}\right)\right] \\
& =\sum_{\sigma_{i-1}}\left[\frac{1}{2}\left(1+\sigma_{i-1}<\sigma_{i-1}>\right) \frac{1}{2}\left(1+<\sigma_{i+1}>\right) \tanh \left(\beta J+\beta J \sigma_{i-1}\right)\right. \\
& =\frac{1}{2} \tanh (2 \beta J)\left[<\sigma_{i-1}>+<\sigma_{i+1}>\right] .
\end{aligned}
$$

A expansão em produtos de spin, que é uma identidade, tem nesse caso a forma 


$$
\tanh \left[\beta J\left(\sigma_{i+1}\right)+\left(\sigma_{i-1}\right)\right]=\frac{1}{2} \tanh (2 \beta J)\left(\sigma_{i+1}+\sigma_{i-1}\right),
$$

que pode ser constatada colocando em $\sigma_{i+1}$ e $\sigma_{i-1}$ todos os seus valores possíveis ( + 1 e -1 para cada um), que dão quatro combinações. O cálculo da média nos dois lados da expressão acima nos conduz ao sistema de equações ( $\mathrm{N}$ equações, para a cadeia de $\mathrm{N}$ spins) que obtivemos anteriormente, mostrando a equivalência dos dois enfoques. Em casos mais complicados a equivalência se mantém se fatorizamos as médias dos produtos de spin que devem aparecer, ou seja, se fazemos sempre $\left\langle\sigma_{j} \sigma_{k}\right\rangle=\left\langle\sigma_{j}\right\rangle\left\langle\sigma_{k}\right\rangle$.

2) Aplicação da aproximação para uma rede $2 \times 2$ ferromagnética.

Numa rede quadrada, com condições de contorno periódicas, um determinado sítio $i$ possui quatro vizinhos. Designando seus vizinhos por $\sigma_{1}, \sigma_{2}, \sigma_{3}$, e $\sigma_{4}$ temos a seguinte identidade ( expansão em produtos de spin):

$$
\begin{aligned}
\tanh \left[\beta J\left(\sigma_{1}+\sigma_{2}+\sigma_{3}+\sigma_{4}\right)\right] & =A_{1}\left(\sigma_{1}+\sigma_{2}+\sigma_{3}+\sigma_{4}\right) \\
& +A_{3}\left(\sigma_{1} \sigma_{2} \sigma_{3}+\sigma_{2} \sigma_{3} \sigma_{4}+\sigma_{3} \sigma_{4} \sigma_{1}+\sigma_{4} \sigma_{1} \sigma_{2}\right),
\end{aligned}
$$

com

$$
A_{1}=\frac{1}{8}[\tanh (4 \beta J)+2 \tanh (2 \beta J)]
$$

e

$$
A_{3}=\frac{1}{8}[\tanh (4 \beta J)-2 \tanh (2 \beta J)]
$$

( Parisi, 1988)

Consideremos agora uma rede quadrada ferromagnética de $2 \times 2$ spins. Nos seus quatro sítios colocamos os spins $\sigma_{1}, \sigma_{2}, \sigma_{3}$, e $\sigma_{4}$. Se usamos condições periódicas de contorno e defini$\operatorname{mos}\left\langle\sigma_{i}\right\rangle=m_{i}=\left\langle\tanh \beta J\left(\sum\right.\right.$ spins vizinhos $\left.\left.a i\right)\right\rangle$ teremos, com fatorização das médias, as equações:

$$
\begin{aligned}
& m_{1}=2 A_{1}\left(m_{3}+m_{2}\right)+2 A_{3}\left(m_{2}^{2} m_{3}+m_{3}^{2} m_{2}\right) \\
& m_{2}=2 A_{1}\left(m_{1}+m_{4}\right)+2 A_{3}\left(m_{1}^{2} m_{4}+m_{4}^{2} m_{1}\right) \\
& m_{3}=2 A_{1}\left(m_{1}+m_{4}\right)+2 A_{3}\left(m_{1}^{2} m_{4}+m_{4}^{2} m_{1}\right)
\end{aligned}
$$

e

$$
m_{4}=2 A_{1}\left(m_{3}+m_{2}\right)+2 A_{3}\left(m_{2}^{2} m_{3}+m_{3}^{2} m_{2}\right) .
$$

Da primeira e da quarta equações temos

$$
m_{1}=m_{4},
$$

da segunda e da terceira,

$$
m_{2}=m_{3}
$$

Em termos de $m_{l}$ e $m_{3}$ ficamos reduzidos às equações 


$$
\begin{aligned}
& m_{3}=a_{1} m_{1}+a_{3} m_{1}^{3} \\
& m_{1}=a_{1} m_{3}+a_{3} m_{3}^{\frac{3}{3}}
\end{aligned}
$$

com $a_{1}=4 A_{1}$ e $a_{3}=4 A_{3}$. Multiplicando a segunda equação por $m_{3}$ e a primeira por $m_{1}$ teremos

$$
a_{1} m_{1}^{2}+a_{3} m_{1}^{4}=a_{1} m_{3}^{2}+a_{3} m_{3}^{4}
$$

que é equivalênte a

$$
\left(m_{1}-m_{3}\right)\left(m_{1}+m_{3}\right)\left[a_{1}+a_{3}\left(m_{1}^{2}+m_{3}^{2}\right)\right]=0
$$

Da expressão acima temos as seguintes possibilidades para relaçōes entre $m_{1}$ e $m_{3}$ :

a) $m_{1}-m_{3}=0$. Fazemos $m_{1}=m$ e $m_{1}=m_{2}=m_{3}=m_{4}$. Esta é a solução de homogeneidade, sendo $m$ dado por

$m=a_{1} m+a_{3} m^{3}$,

que é uma equação com as soluções $m=0$ e $m= \pm \sqrt{\frac{\left(1-a_{1}\right)}{a_{3}}}$. A solução $m \neq 0$ é possível na medida em que

$1-a_{1}<0$, dado que $a_{3}<0$ sempre, implicando que

$\frac{1}{2}[\tanh (4 \beta J)+2 \tanh (2 \beta J)]>1$,

que é uma relação válida se

$T=\frac{1}{\beta J}<3.0898 \ldots$.

b) $m_{1}=-m_{3}=M$, levando à equação $M=-a_{1} M-a_{3} M^{3}$,

com soluçōes $M=0 \mathrm{e}$

$M= \pm \sqrt{\frac{-\left(1+a_{1}\right)}{a_{3}}}$.

Porém, por substituição dos valores de $a_{1}$ e $a_{3}$, observamos que a solução com $M \neq 0$ dá M > 1 para todos os valores de $T$, e deve ser descartada

c) $a_{1}+a_{3}\left(m_{1}^{2}+m_{3}^{2}\right)=0$. Substituindo em $m_{3}=a_{1} m_{1}+a_{3} m_{1}^{3}$,

que é a primeira de nossas equações originais, teremos a equação

$m_{3}^{4}+\frac{a_{1}}{a_{3}} m_{3}^{2}+\frac{1}{a_{3}^{2}}=0$

Entretanto, uma vez que 
$\left(\frac{a_{1}}{a_{3}}\right)^{2}-\frac{4}{a_{3}^{2}}=\frac{a_{1}^{2}-4}{a_{3}}<0$

para todos os valores de $T$, não há chance de obtermos soluções reais nesse caso.

Do que dissemos acima, podemos ver que para uma rede $2 \times 2$, nesta aproximação, o valor da temperatura de Curie é 3.0898... . O mesmo acontece para uma rede de qualquer tamanho onde aplicamos de imediato a homogeneidade. Se, por exemplo, tratamos uma rede ferromagnética dividindo-a em duas subredes, caimos num sistema de duas equações como as já discutidas, e somos levados a acreditar que numa rede quadrada ferromagnética a consideração de inomogeneidade não leva a soluções plausíveis. Devemos lembrar que esse resultado para a temperatura de Curie já era conhecido pelo menos desde 1968, por Mamada e Takano ( Mamada, 1968 ). 


\section{Apêndice 3.}

Prova de equivalência entre as abordagens de Correlação Temporal e de Ineficiência Estatística para a análise de erros num processo Monte Carlo (Henriques, 1992).

A análise de erros numa simulação de Monte Carlo se complica devido às correlações entre as configurações MC sucessivas que são geradas no processo, o que impede o uso de fórmulas convencionais de avaliação de erros. Uma das maneiras de contornarmos o problema é pelo cálculo da variância das médias sobre blocos de sucessivas configurações Monte Carlo de tamanho $P$. Existe um limite superior para $P$ a partir do qual as médias nos blocos podem ser consideradas como dados estatisticamente independentes e mostraremos aqui que a procura por esse limite corresponde à análise de correlação temporal, como mostrada por Binder e Heermann ( Binder, 1988 ).

Suponhamos uma sequência de $M$ dados. Definimos:

a) blocos de tamanho $P$ que dividem $M$ em $M_{p}$ blocos, com $M=P M P$

b) a média no bloco $i$ de uma grandeza $X$ é

$\bar{X}=\frac{1}{P} \sum_{j=1}^{P} X_{j}^{i}$,

sendo $j$ o índice das $P$ configurações sucessivas dentro do bloco $i$.

c) A média das médias, que é também a média dos $M$ dados é

$\bar{X}=\frac{1}{M_{P}} \sum_{i=1}^{M_{P}} \bar{X}_{i}$.

d) A variância total dos $M$ dados é

$$
\sigma^{2}=\frac{1}{M} \sum_{i=1}^{M P} \sum_{j=1}^{P}\left(X_{j}^{i}-X\right)^{2}
$$

e) A variância das médias nos blocos de tamanho $P$ é

$$
\sigma_{P}^{2}=\frac{1}{M_{P}} \sum_{i=1}^{M_{P}}\left(\overline{X_{i}}-\bar{X}\right)^{2}
$$

Vamos desenvolver a expressão de $\sigma \mathfrak{P}$. Temos

$$
\begin{aligned}
\sigma_{P}^{2} & =\frac{1}{M_{P}} \sum_{i=1}^{M_{P}}\left(\overline{X_{i}}-\bar{X}\right)^{2} \\
& =\frac{1}{M_{P}} \sum_{i=1}^{M_{P}}\left(\overline{X_{i}}\right)^{2}-\frac{1}{M_{P}} \sum_{i=1}^{M_{P}} 2 \bar{X}_{i} X+\frac{1}{M_{P}} \sum_{i=1}^{M_{P}}(X)^{2}
\end{aligned}
$$




$$
\begin{aligned}
& =\frac{1}{M_{P}} \sum_{i=1}^{M_{P}}\left(\overline{X_{i}}\right)^{2}-2 X\left(\frac{1}{M_{P}} \sum_{i=1}^{M_{P}} \overline{X_{i}}\right)+(\bar{X})^{2} \frac{1}{M_{P}} \sum_{i=1}^{M_{P}} 1 \\
& =\left[\frac{1}{M_{P}} \sum_{i=1}^{M_{P}}\left(\overline{X_{i}}\right)^{2}\right]-(\bar{X})^{2} \\
& =\frac{1}{M_{P}} \sum_{i=1}^{M_{P}}\left[\left(\overline{X_{i}}\right)^{2}-(\bar{X})^{2}\right] \\
& =\frac{1}{M_{P}} \sum_{i=1}^{M_{P}} \delta_{i}^{2}
\end{aligned}
$$

com $\delta_{i}^{2}=\left(\bar{X}_{i}\right)^{2}-(\bar{X})^{2}$. Podemos escrever

$$
\begin{aligned}
\left(X_{i}\right)^{2} & =\frac{1}{P^{2}}\left(\sum_{j=1}^{P} X_{j}^{i}\right)\left(\sum_{k=1}^{P} X_{k}^{i}\right) \\
& =\frac{1}{P^{2}}\left[\sum_{j=1}^{P}\left(X_{j}^{i}\right)^{2}+\sum_{j=1}^{P} \sum_{k=1 \neq j}^{P} X_{j}^{i} X_{k}^{j}\right] \\
& =\frac{1}{P^{2}}\left\{\sum_{j=1}^{P}\left[\left(X_{j}^{i}\right)^{2}-(X)^{2}\right]+P(X)^{2}+\sum_{j=1}^{P} \sum_{k=1 \neq j}^{P}\left[X_{j}^{i} X_{k}^{i}-(X)^{2}\right]+P(P-1)(X)^{2}\right\},
\end{aligned}
$$

de forma que

$$
\begin{aligned}
& \delta_{i}^{2}=\left(X_{i}\right)^{2}-(X)^{2} \\
= & \frac{1}{P^{2}}\left\{\sum_{j=1}^{P}\left[\left(X_{j}^{i}\right)^{2}-(X)^{2}\right]+\sum_{j=1}^{P} \sum_{k=1 \neq j}^{P}\left[X_{j}^{i} X_{k}^{i}-(X)^{2}\right]\right. \\
& \left.+P(\bar{X})^{2}+P^{2}(\bar{X})^{2}-P(\bar{X})^{2}\right\}-(\bar{X})^{2} .
\end{aligned}
$$

Assim sendo

$$
\begin{aligned}
\sigma^{2}= & \frac{1}{M_{P}} \sum_{i=1}^{M_{P}} \delta_{i}^{2} \\
& =\frac{1}{M_{P} P^{2}} \sum_{i=1}^{M_{P}} \sum_{j=1}^{P}\left[\left(X_{j}^{i}\right)^{2}-(X)^{2}\right]+\frac{1}{M_{P} P^{2}} \sum_{i=1}^{M_{P}} \sum_{j=1}^{P} \sum_{k=1 \neq j}^{P}\left[X_{j}^{i} X_{k}^{j}-(X)^{2}\right],
\end{aligned}
$$

com o que

$$
\sigma^{2}=\frac{\sigma^{2}}{P}+\frac{1}{M P P^{2}} \sum_{i=1}^{M_{P}} \sum_{j=1}^{P} \sum_{k=1 \neq j}^{P}\left[X_{j}^{i} X_{k}^{i}-(X)^{2}\right]
$$

pois 


$$
\begin{aligned}
\sigma^{2} & =\frac{1}{M} \sum_{i=1}^{M P} \sum_{j=1}^{P}\left(X_{j}^{i}-\bar{X}\right)^{2} \\
& =\frac{1}{P M P} \sum_{i=1}^{M P} \sum_{j=1}^{P}\left[\left(X_{j}^{i}\right)^{2}-2 X_{j}^{i}(\bar{X})+(\bar{X})^{2}\right] \\
& =\frac{1}{P M P} \sum_{i=1}^{M P} \sum_{j=1}^{P}\left[\left(X_{j}^{i}\right)^{2}-(\bar{X})^{2}\right]
\end{aligned}
$$

Podemos observar que $\sigma^{2} \not$ se divide em dois termos: $\frac{\sigma^{2}}{P}$ e um termo que representa a soma, para todos os $M P$ blocos, dos termos de correlação dentro de cada bloco. Se temos uma coleção de $P$ dados estatisticamente independentes, $\sigma_{P}^{2}=\frac{\sigma^{2}}{P}$ dá o erro estatístico da medida com os $P$ dados, onde $\sigma^{2}$ é avaliado a partir desses dados (Helene, 1981). Porém, em Monte Carlo isso não ocorre, e nem mesmo a soma dos termos de correlação para os $M P$ blocos se anula.

É interessante reescrever $\sigma^{2}$ na forma de correlações "temporais" de pares. Nosso objetivo é a comparação do que estamos fazendo aqui com a análise de correlações temporais no processo (Binder, 1988).

Observemos, primeiramente, que

$$
\sum_{j=1}^{P} \sum_{k=1 \neq j}^{P} X_{j}^{i} X X_{k}^{i}=2 \sum_{j=1}^{P-1} \sum_{k=1}^{P-j} X \dot{k}_{k} X \dot{k}_{k+j}
$$

Introduzimos agora a média dentro do bloco $i$ dos produtos de pares $X_{k}^{i}$ e $X \dot{k}+j$ que são próximos entre si de uma "distância" ( ou "intervalo de tempo") $j$, de forma que $j=1$ representa valores sucessivos, $j=2$ valores sucessivos pulando um valor e assim por diante. Essa média é

$$
<X_{k} X_{k+j}>^{i}=\frac{1}{P-j} \sum_{k=1}^{P-j} X_{k}^{j} X \dot{k}_{k+j}
$$

e é a covariância dos pares de $X$ que "distam" $j$ valores entre si. Com essa definição

$$
\sum_{j=1}^{P} \sum_{k=1 \neq j}^{P} X_{j}^{i} X_{k}^{i}=2 \sum_{j=1}^{P-1}(P-j)\left\langle X_{k} X_{k+j}\right\rangle^{i}
$$

Podemos, além disso, escrever

Dessa forma, temos

$$
\sum_{j=1}^{P} \sum_{k=1 \neq j}^{P}(X)^{2}=P(P-1)(X)^{2}=2 \sum_{j=1}^{P-1}(P-j)(X)^{2} .
$$

$$
\sum_{j=1}^{P} \sum_{k=1 \neq j}^{P}\left[X_{j}^{i} X_{k}^{j}-(X)^{2}\right]=2 \sum_{j=1}^{P-1}(P-j)\left[\left\langle X_{k} X_{k+j}\right\rangle^{i}-(X)^{2}\right],
$$


e podemos expressar $\sigma^{2}$ como

$$
\sigma^{2}=\frac{\sigma^{2}}{P}+\frac{2}{M P P^{2}} \sum_{i=1}^{M_{P}} \sum_{j=1}^{P-1}(P-j)\left[\left\langle X_{k} X_{k+j}\right\rangle^{i}-(X)^{2}\right] .
$$

Vamos agora definir a função de correlação de pares $X$ que "distam" $j$ configurações entre si, dentro de nossa amostragem de $M$ dados e dada uma partição de $M P$ blocos de tamanho $P$, como sendo

$$
C_{X}{ }^{P}(j)=\frac{\frac{1}{M_{P}} \sum_{i=1}^{M_{P}}\left[\left\langle X_{k} X_{k+j}\right\rangle^{i}-(\bar{X})^{2}\right]}{\sigma^{2}}
$$

$\mathrm{Na}$ expressão acima, o numerador representa a média de $M_{p}$ valores da correlação dos pares que "distam" $j$ entre si em cada bloco $i$, isto é, fazemos bloco a bloco essa média ( que é a correlação no bloco) e tomamos as $M_{p}$ médias. Desde que não há produtos $X_{k} X_{k+j}$ fora de um bloco $i$ em nossa definição de correlação, devemos encarar $C_{X}{ }^{P}(j)$ como um estimador da correlação de pares de $X$ que distam $j$ valores entre si na sequência de $M$ valores . Esse estimador será tanto pior quanto maior for o valor de $j$. Com essa definição escrevemos

$$
\begin{aligned}
\sigma_{P}^{2} & =\frac{\sigma^{2}}{P}+\frac{2}{M_{P} P^{2}} \sum_{i=1}^{M_{P}} \sum_{j=1}^{P-1}(P-j)\left[\left\langle X_{k} X_{k+j}\right\rangle^{i}-(X)^{2}\right] \\
& =\frac{\sigma^{2}}{P}+\frac{2}{M_{P} P^{2}} \sum_{j=1}^{P-1} \sum_{i=1}^{M_{P}}(P-j)\left[\left\langle X_{k} X_{k+j}\right\rangle^{i}-(\bar{X})^{2}\right] \\
& =\frac{\sigma^{2}}{P}+\frac{2}{M_{P} P^{2}} \sum_{j=1}^{P-1}(P-j) \sum_{i=1}^{M}\left[\left\langle X_{k} X_{k+j}\right\rangle^{i}-(\bar{X})^{2}\right] \\
& =\frac{\sigma^{2}}{P}+\frac{2}{M P P^{2}} \sum_{j=1}^{P-1}(P-j) \sigma^{2} C_{X} P(j) M_{P} \\
& =\frac{\sigma^{2}}{P}\left\{1+2 \sum_{j=1}^{P-1}\left(1-\frac{j}{P}\right) C_{X}^{P}(j)\right\} .
\end{aligned}
$$

Temos agora o que queríamos, ou seja, temos $\sigma^{2} P$ expresso em termos da função de correlação "temporal". Esperamos que para $P$ suficientemente grande, a somatória da expressão acima tenderá a um limite definido por $\tau_{\mathrm{A}}$, ou seja, esperamos que

$$
\tau_{A}=\lim _{P \rightarrow \infty} \sum_{j=1}^{P-1}\left(1-\frac{j}{P}\right) C_{X}{ }^{P}(j)
$$

seja um valor finito. Para observar isso vamos imaginar uma forma exponencial para $C_{X}{ }^{P}(j)$ 
(Gould e Tobochnik, Ref.25). Passsando ao limite do contínuo, o índice $j$ se transforma em $t$ ( de tempo), e teremos

$$
\begin{aligned}
\Delta(P) & \equiv \sum_{j=1}^{P-1}\left(1-\frac{j}{P}\right) C_{k}^{P}(j) \\
& \approx \int_{0}^{P}\left(1-\frac{t}{P}\right) e^{-\frac{t}{\tau_{A}}} d t=\tau_{A}\left[1-\frac{\tau_{A}}{P}\left(1-e^{-\frac{P}{\tau_{A}}}\right)\right] .
\end{aligned}
$$

De fato, observamos que

$$
\lim _{P \rightarrow \infty} \Delta(P)=\tau_{A},
$$

de maneira que

$$
\sigma_{P}^{2}=\frac{\sigma^{2}}{P}[1+2 \Delta(P)]
$$

tenderá, quando $P>>\tau_{A}$, para

$$
\sigma_{P}^{2}=\frac{\sigma^{2}}{P}\left[1+2 \tau_{A}\right] \text {. }
$$

A grandeza $I(P)$ do capítulo II foi definida como

$$
I(P)=\frac{\frac{\sigma^{2}}{M P}}{\frac{\sigma^{2}}{M}} .
$$

Segundo o que dissemos mais acima temos

$$
I(P)=\frac{M}{\sigma^{2}} \frac{\sigma^{2}}{P M P}[1+2 \Delta(P)]=1+2 \Delta(P)
$$

Se observamos a expressão de $\Delta(P)$, vemos que ela é uma função crescente de $P$, com

$$
\lim _{P \rightarrow 0} \Delta(P)=0 \quad \text { e com } \lim _{P \rightarrow \infty} \Delta(P)=\tau_{A} .
$$

Confirmando o que dissemos no capítulo II sobre o comportamento de $I(P)$ é crescente com $P$ e se estaciona para um valor limite de $P$, chamado de ineficiência estatística. Então

$$
\text { Ineficiência Estatística }=I=1+2 \tau_{A},
$$

e o cálculo da ineficiência é equivalente à análise de correlações temporais.

Observemos, por final, que o erro $\varepsilon$ na medida da grandeza $X$, tomada em $M$ observações sucessivas $\mathrm{X}_{\mu}$, com $\mu=1,2, \ldots M$ denotando as configurações (passos de Monte Carlo) é definido por

$$
\varepsilon^{2}=\left\langle\left[\frac{1}{M} \sum_{\mu=1}^{M}\left(X_{\mu}-<X>\right)\right]^{2}>\right.
$$

e prova-se (Binder, 1988) que quando $M$ é grande

$$
\varepsilon^{2}=\frac{1}{M}\left[\left\langleX^{2}>-\left\langle X>^{2}\right]\left(1+2 \tau_{A}\right)=\frac{\sigma^{2}}{M}\left(1+2 \tau_{A}\right),\right.\right.
$$


Assim, em nossa linguagem dos blocos, o erro deve ser calculado, dado $\sigma_{P}^{2}$, fazendo-se

$$
\varepsilon^{2}=\frac{\sigma P_{\max }}{M P_{\text {max }}}
$$

onde $P_{\max }$ é o limite em que podemos considerar que $\Delta(P)$ está estabilizado nas proximidades de $\tau_{A}$ Da expressão acima para $\varepsilon^{2}$, podemos interpretar as $M_{P_{\text {máx }}}$ médias nos blocos como dados estatísticamente independentes, como está dito no capítulo II. 
Forma simplificada do Algoritmo de Netz e Berker para um Modelo de Ising numa rede quadrada. Do lado direito estão algumas explicações auxiliares.

\section{< Início}

Variáveis inteiras cont, $k$,desc, I, j, la, ja, Ip, jp, N

Variáveis reais magk, mag, $\mathrm{t}$

Matrizes reais $\mathrm{s}(30,30)$, $\mathrm{m}(30,30)$

\section{Faça \\ $\ll \mathrm{N}=30$ \\ cont $=500$ \\ $\operatorname{desc}=100$ \\ $\operatorname{mag}=0.0$ \\ $t=0.2$}

para $\mathrm{I}=1$ até $\mathrm{N}$ e $\mathrm{j}=1$ até $\mathrm{N}$ repita

$\ll m(1, j)=1.0 \gg$

para $k=\uparrow$ até cont repita

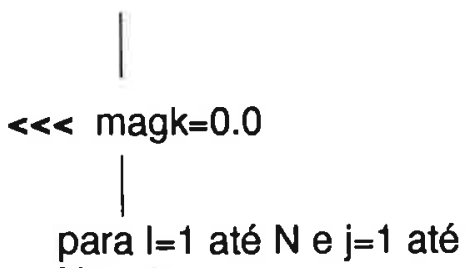

$\mathrm{N}$ repita<smiles>CC[As](C)C</smiles>

$j a=j-1$

$|p=|+1$

j $p=j+1$

Se la=0 então $\mathrm{la}=\mathrm{N}$
*Estamos declarando as variáveis. Haverá cont PMCs, contados com o índice $k$, e dos quais descartamos os desc iniciais. I e j localizam um sítio e la, ja, Ip. jp são auxiliares na aplicação das condições de contorno periódicas. $N$ é um lado da rede, em no de spins, magk é o valor da magnetização por spin no k-ésimo passo e mag é a média para todos os passos contados. té a temperatura em unidades de $1 / \beta \mathrm{J}$ e s e m são as matrizes onde guardamos os spins $\theta$ os valores de $\tanh \left[\beta \mathrm{J}\left(\sum\right.\right.$ spins vizinhos a $\left.\left.(\mathrm{l}, \mathrm{j})\right)\right]$

*A rede possuirá 900 spins ( e ms )

*500 PMC

*400 passos para a média

*Inicializamos a média

*Fixamos o valor de t

*Inicialização dos valores de ms

*Cada k representa um PMC, do qual saímos com uma nova configuração de $\mathrm{ms}$

*Inicialização do valor de magnetização por spin no passo $\mathrm{k}$

*Fixação das condições de contorno periódicas 
Se $\mathrm{ja}=0$ então $\mathrm{ja}=\mathrm{N}$

Se $\mid p=N+1$ então $\mid p=1$

Se $j p=N+1$ então j $p=1$

$s(l a, j)=$ sinal de $(\mathrm{m}(\mathrm{la}, \mathrm{j})$ - rand $)$

$s(I p, j)=$ sinal de

( $\mathrm{m}(\mathrm{l}, \mathrm{ja})$ - rand )

$s(\mathrm{l}, \mathrm{ja})=$ sinal de

$(\mathrm{m}(\mathrm{l}, \mathrm{ja})$ - rand $)$

$s(I, j p)=$ sinal de

( $m(I, j p)$ - rand )

$m(I, j)=$

$\tanh [(\mathrm{s}(\mathrm{la}, \mathrm{j})+$

$s(l p, j)+s(l, j a)+$ $s(I, j p)) / t]$

$\gg>$

seké maior do que desc faça

* atualização do valor de m no sítio de coordenadas $(I, j)$

*Aplicação da prescrição para reconstrução do campo no sítio de coordenadas $(I, j)$, com rand entre $-1 e+1$

* fechamento do loop de atualização dos ms

*a partir do ponto em que o № de PMC é maior que desc acumulamos em mag o valor de magk que obtemos pela soma dos ms. Quando k=cont, o loop é fechado. Se quizermos, poderemos acumular os valores de outra grandeza.

$\ll$ para $\mid=1$ até $\mathrm{N}$

$\mathrm{e} \mathrm{j}=1$ até $\mathrm{N}$ repita

«

magk $+m(1, j)$

$\gg>>$

mag=

$\operatorname{mag}+\operatorname{magk} /\left(N^{\star} N\right)$

$\gg>$

>>

mag $=\mathrm{mag} /($ cont - desc $)$

*cálculo e impressão da média

imprima mag $\gg>$

Fim >

Nesta versão simplificada, não explicitamos a função rand e não fizemos tabelas para os cálculos dos mi. Ela serve apenas como uma indicação do que foi feito. Lembramos que a função rand deve devolver números aleatórios entre -1 e +1 . 


\section{Referências}

Arora, B.L e Landau,D.P. (1971), AIP conf. Proc. no 5 (AIP. New York), p. 352.

Banavar, Cieplak e Maritan (1991), Phys. Rev. lett $67,1807$.

Binder, K. (1981), Z.Phys. B 45, 61.

e Heermann, D.W. (1988), "Monte Carlo Simulation in Statistical Physics (An Introduction)", Springer Verlag.

Blume, M. (1966), Physical Review $141,517$.

, Emery, V.J. e Griffiths, R.B. (1971) Phys. rev. A 4 , 1071.

Brush, S.G. (1983), "Statistical Physics and the Atomic Theory of Matter from boyle and Newton to Landau and Onsager", Princeton.

Callen.H. (1985), "Thermodynamics and an Introduction to Thermostatisties, Second Edition", Wiley.

Capel, H. W. (1966), Physica $32,966$.

Friedberg e Cameron (1970), Journal of Chemical Physics 52, 6049.

Glauber, R.J. (1963), Journal of Mathematical Physics 4 (2), 294.

Gould, H. e Tobochnik, F. (1989), Computers in Physics 3 (4), 82.

Helene, O. A. M.e Vauin, V. (1981), "Tratamento estatístico de dados em Física Experimental", Edgard Bücher.

Henriques, Vera B. (1992), comunicação pessoal.

Kittel, C. (1967), "Introduction to Solid State Physics", Wiley.

Landau, D. P. (1976), Phys. Rev. B 13, 2997.

Mamada, H. e Takano, F. (1968), Journal of the Physical Society of Japan 25, 675.

Netz, R. e Berker, A.N. (1991 a), Phys. Rev. Lett. 66 , 337.

(1991 b), Phys. Rev. Lett. $67,1808$.

(1991 c), J. Appl. Phys. 70 , 6074.

Oliveira, M. J. e Salinas, S. R. (1985), Revista Brasileira de Física 15 , 189.

Parisi, G. (1988), "Statistical Field Theory", Addison Wesley.

Stanley, H.E. (1971), "Introduction to Phase Transitions and Critical Phenomena", Oxford.

Thompson, C. J. (1988), "Classical Equilibrium Statistical Mechanics", Oxford. 